\title{
THE HARMONIC MEAN CURVATURE FLOW OF NONCONVEX SURFACES IN $\mathbb{R}^{3}$
}

\author{
PANAGIOTA DASKALOPOULOS* AND NATASA SESUM*
}

\begin{abstract}
We consider a compact star-shaped mean convex hypersurface $\Sigma^{2} \subset \mathbb{R}^{3}$. We prove that in some cases the flow exists until it shrinks to a point. We also prove that in the case of a surface of revolution which is star-shaped and mean convex, a smooth solution always exists up to some finite time $T<\infty$ at which the flow shrinks to a point asymptotically spherically.
\end{abstract}

\section{INTRODUCTION}

We will consider in this work the deformation of a compact hyper-surface $\Sigma_{t}$ in $\mathbb{R}^{3}$ with no boundary under the harmonic mean curvature flow (HMCF) namely the flow

$$
\frac{\partial P}{\partial t}=-\frac{G}{H} \nu
$$

which evolves each point $P$ of the surface in the direction of its normal unit vector with speed equal to the harmonic mean curvature of the surface $G / H$, with $G$ denoting the Gaussian curvature of $\Sigma_{t}$ and $H$ its mean curvature. Here $\nu$ denotes the outer unit normal to the surface at $P$. This flow remains weakly parabolic without the condition that $\Sigma_{t}$ is strictly convex. However, it becomes degenerate at points where the Gaussian curvature $G$ vanishes.

The existence of solutions to the HMCF with strictly convex smooth initial data was first shown by Andrews in [3] who also showed that under the HMCF strictly convex smooth surfaces converge to round points in finite time. In 8 , Diëter established the short time existence of solutions to the HMCF with weakly convex smooth initial data. More precisely, Dieter showed that if at time $t=0$ the surface $\Sigma_{0}$ satisfies $G \geq 0$ and $H>0$, then there exists a unique strictly convex smooth solution $\Sigma_{t}$ of the HMCF defined on $0<t<\tau$, for some $\tau>0$. By the results of Andrews, the solution will exist up to the time where its enclosed volume becomes zero.

* : Partially supported by NSF grant 0604657. 
In [5] Caputo and the first author considered the highly degenerate case where the initial surface is weakly convex with flat sides, where the parabolic equation describing the motion of the surface becomes highly degenerate at points where both curvatures $G$ and $H$ become zero. The solvability and optimal regularity of the surface $\Sigma_{t}$, for $t>0$, was addressed and studied by viewing the flow as a freeboundary problem. It was shown that a surface $\Sigma_{0}$ of class $C^{k, \gamma}$ with $k \geq 1$ and $0<\gamma \leq 1$ at $t=0$, will remain in the same class for $t>0$. In addition, the strictly convex parts of the surface become instantly $C^{\infty}$ smooth up to the flat sides on $t>0$, and the boundaries of the flat sides evolve by the curve shortening flow.

The case $G<0$ was recently studied by the first author and R. Hamilton in [7, under the assumption that the initial surface is a surface or revolution with boundary, and has $G<0$ and $H<0$ everywhere. It was shown in [7] that under certain boundary conditions, there exists a time $T_{0}>0$ for which the HMCF admits a unique solution $\Sigma_{t}$ up to $T_{0}$, such that $H<0$ for all $t<T_{0}$ and $H\left(\cdot, T_{0}\right) \equiv 0$ on some set of sufficiently large measure. In addition, the boundary of the surface evolves by the curve shortening flow.

In this work we address the questions of short time and long time existence and regularity of the HMCF under the assumption that $\Sigma_{0}$ is star-shaped with $H>0$ but with $G$ changing sign.

Let $M^{2}$ be a smooth, compact surface without boundary and $F_{0}: M^{2} \rightarrow \mathbb{R}^{3}$ be a smooth immersion of $M^{2}$. Let us consider a smooth family of immersions $F(\cdot, t): M^{2} \rightarrow \mathbb{R}^{3}$ satisfying

$$
\frac{\partial F(p, t)}{\partial t}=-\kappa(p, t) \cdot \nu(p, t)
$$

where $\kappa=G / H$ denotes the harmonic mean curvature of $\Sigma_{t}:=F\left(M^{2}, t\right)$ and $\nu$ its outer unit normal at every point. This is an equivalent formulation of the HMCF.

For any compact two-dimensional surface $M^{2}$ which is smoothly embedded in $\mathbb{R}^{3}$ by $F: M^{2} \rightarrow \mathbb{R}^{3}$, let us denote by $g=\left(g_{i j}\right)$ the induced metric, and by $\nabla$ the induced Levi-Civita connection. The second fundamental form $A=\left\{h_{i j}\right\}$ is a symmetric bilinear form $A(p): T_{p} \Sigma \times T_{p} M \rightarrow \mathbb{R}$, defined by $A(u, v)=\left\langle\nabla_{u} \nu, v\right\rangle$. The Weingarten map $W(p): T_{p} \Sigma \rightarrow T_{p} \Sigma$ of $T_{p} M$ given by the immersion $F$ with respect to the normal $\nu$, can be computed as $h_{j}^{i}=g^{i k} h_{k j}$. The eigenvalues of $W(p)$ are called the principal curvatures of $F$ at $p$ and are denoted by $\lambda_{1}=\lambda_{1}(p)$ and $\lambda_{2}=\lambda_{2}(p)$. The mean curvature $H:=\operatorname{trace}(W)=\lambda_{1}+\lambda_{2}$, the total curvature $|A|^{2}:=\operatorname{trace}\left(W^{t} W\right)=\lambda_{1}^{2}+\lambda_{2}^{2}$ and the Gauss curvature $G=\operatorname{det} W=\lambda_{1} \lambda_{2}$. 
Remark 1.1. We will recall some standard facts about homogeneous of degree one functions of matrices that can be found in [1]. The speed speed $\kappa$ of the interface evolving by the HMCF can be viewed as a function of the Weingarten map $W$ and therefore, more generally, as a function $\kappa: S \rightarrow \mathbb{R}$, where $S$ denotes the set of all symmetric, positive transformations of $T \Sigma^{2}$ with strictly positive trace. Let $\lambda_{1}, \lambda_{2}$ be the eigenvalues of $A \in S$. We can then define the symmetric function $f\left(\lambda_{1}, \lambda_{2}\right):=\kappa(A)$. We have:

- If $f$ is concave (convex) and $\lambda_{i}>\lambda_{j}$, then $\frac{\partial f}{\partial \lambda_{i}}-\frac{\partial f}{\partial \lambda_{j}}$ is negative (positive).

- Let $\ddot{\kappa} \in T \Sigma \otimes T^{*} \Sigma \otimes T \Sigma \otimes T^{*} \Sigma$ denote the second derivative of $\kappa$ at the point $A \in S$. If $A$ is diagonal, then

$$
\ddot{\kappa}(\xi, \eta)=\sum_{p, q} \frac{\partial^{2} \kappa}{\partial \lambda_{p} \lambda_{q}} \xi_{p}^{p} \eta_{q}^{q}+\sum_{p \neq q} \frac{\frac{\partial \kappa}{\partial \lambda_{p}}-\frac{\partial \kappa}{\partial \lambda_{q}}}{\lambda_{p}-\lambda_{q}} \xi_{p}^{q} \eta_{p}^{q} .
$$

The outline of the paper is as follows:

i. In section 2 we will establish the short time existence of (HMCF), under the assumption that the initial surface $\Sigma_{0}$ is compact of class $C^{2,1}$ and has $H>0$. To do so we will have to bound $H$ from below away from zero independently of $\epsilon$. This does not follow naturally from the evolution of $H$. To obtain such a bound we need to combine the evolution of $H$ with the evolution of the gradient of the second fundamental form. This explains our assumption that $\Sigma \in C^{2,1}$.

ii. In section 3 we will study the long time existence of the regularized flow $\left(\mathrm{HMCF}_{\epsilon}\right)($ defined in the next section). We will show that there exists a maximal time of existence $T_{\epsilon}$ of a smooth solution $\Sigma_{t}^{\epsilon}$ of $\left(\mathrm{HMCF}_{\epsilon}\right)$ such that either $H\left(P_{t}, t\right) \rightarrow 0$, as $t \rightarrow T_{\epsilon}$ at some points $P_{t} \in \Sigma_{t}^{\epsilon}$, or $\Sigma_{t}^{\epsilon}$ shrinks to a point as $t \rightarrow T_{\epsilon}$. In addition, we will establish uniform in $\epsilon$ curvature bounds and curvature pinching estimates. In the special case where the initial data is a surface of revolution, we will show that the flow always exists up to the time when the surface shrinks to a point.

iii. In section 4 we will pass to the limit, $\epsilon \rightarrow 0$, to establish the long time existence of (HMCF).

\section{Short time Existence}

Our goal in this section is to show the following short time existence result for the HMCF. 
Theorem 2.1. Let $\Sigma_{0}$ be a compact hyper-surface in $\mathbb{R}^{3}$ which is of class $C^{2,1}$ and has strictly positive mean curvature $H>0$. Then, there exists $T>0$ for which the harmonic mean curvature flow (HMCF) admits a unique $C^{2,1}$ solution $\Sigma_{t}$, such that $H>0$ on $t \in[0, T)$.

Because the harmonic mean curvature flow becomes degenerate when the Gauss curvature of the surface $\Sigma_{t}$ changes sign, we will show the short time existence for equation (HMCF) by considering its $\epsilon$-regularization of the flow defined by

$$
\frac{\partial F_{\epsilon}}{\partial t}=-\left(\frac{G}{H}+\epsilon H\right) \cdot \nu
$$

and starting at $\Sigma_{0}$. We will denote by $\Sigma_{t}^{\epsilon}$ the surfaces obtained by evolving the initial surface $\Sigma_{0}$ along the flow $\left(\mathrm{HMCF}_{\epsilon}\right)$.

Since the right hand side of $\left(\overline{\mathrm{HMCF}_{\epsilon}}\right)$ can be viewed as a function of the second fundamental form matrix $A$, a direct computation shows that its linearization is given by

with

$$
\mathcal{L}_{\epsilon}(u)=\frac{\partial}{\partial h_{k}^{i}}\left(\frac{G}{H}+\epsilon H\right) \nabla_{i} \nabla_{k} u=a_{\epsilon}^{i k} \nabla_{i} \nabla_{k} u
$$

Notice that if we compute $a_{\epsilon}^{i k}$ in geodesic coordinates around the point (at which the matrix $A$ is diagonal) we get

$$
a_{\epsilon}^{i k}=\left(\begin{array}{cc}
\frac{\lambda_{2}^{2}}{\left(\lambda_{1}+\lambda_{2}\right)^{2}}+\epsilon & 0 \\
0 & \frac{\lambda_{1}^{2}}{\left(\lambda_{1}+\lambda_{2}\right)^{2}}+\epsilon
\end{array}\right)
$$

which is strictly positive definite, no matter what the principal curvatures are.

The following short time existence for the regularized flow $\left(\mathrm{HMCF}_{\epsilon}\right)$ follows from the standard theory on the existence of solutions to strictly parabolic equations.

Proposition 2.2. Let $\Sigma_{0}$ be a compact hyper-surface in $\mathbb{R}^{3}$ which is of class $C^{1,1}$ and has strictly positive mean curvature $H>0$. Then, there exists $T_{\epsilon}>0$, for which the harmonic mean curvature flow $\left(H M C F_{\epsilon}\right)$ admits a smooth solution $\Sigma_{t}^{\epsilon}$, such that $H>0$ on $t \in\left[0, T_{\epsilon}\right)$.

Our goal is to show that if the initial surface $\Sigma_{0}$ is of class $C^{2,1}$, then there is a $T_{0}>0$, so that $T_{\epsilon} \geq T_{0}$ for every $\epsilon$ and that we have uniform estimates on $F_{\epsilon}$, independent of $\epsilon$, so that we can take a limit of $F_{\epsilon}$ as $\epsilon \rightarrow 0$ and obtain a solution of (HMCF that is of class $C^{2,1}$. The main obstacle here is to exclude that 
THE HARMONIC MEAN CURVATURE FLOW OF NONCONVEX SURFACES IN $\mathbb{R}^{3} 5$

$H_{\epsilon}\left(P_{\epsilon}, t_{\epsilon}\right) \rightarrow 0$, as $\epsilon \rightarrow 0$, for some points $P_{\epsilon} \in \Sigma_{t_{\epsilon}}^{\epsilon}$ and times $t_{\epsilon} \rightarrow 0$. Notice that our flow cannot be defined at points where $H=0$.

\section{Notation.}

- When there is no possibility of confusion, we will use the letters $c, C$ and $T_{0}$ for various constants which are independent of $\epsilon$ but change from line to line.

- Throughout this section we will denote by $\lambda_{1}, \lambda_{2}$ the two principal curvatures of the surface $\Sigma_{t}^{\epsilon}$ at a point $P$ and will assume that $\lambda_{1} \geq \lambda_{2}$.

- When there is no possibility of confusion we will drop the index $\epsilon$ from $H, G, A, g_{i j}, h_{i j}$ etc.

The next lemma follows directly from the computations of B. Andrews in [1] (Chapter 3).

Lemma 2.3. If $\Sigma_{t}^{\epsilon}$ moves by $\left(H M C F_{\epsilon}\right)$, with speed $\kappa_{\epsilon}:=\frac{G}{H}+\epsilon H$, the computation in 1 gives us the evolution equations

i. $\frac{\partial}{\partial t} H=\mathcal{L}_{\epsilon} H+\frac{\partial^{2} \kappa_{\epsilon}}{\partial h_{q}^{p} \partial h_{m}^{l}} \nabla^{i} h_{q}^{p} \nabla_{j} h_{m}^{l}+\frac{\partial \kappa_{\epsilon}}{\partial h_{m}^{l}} h_{p}^{l} h_{m}^{p} H$

ii. $\frac{\partial}{\partial t} \kappa_{\epsilon}=\mathcal{L}_{\epsilon} \kappa_{\epsilon}+\frac{\partial \kappa_{\epsilon}}{\partial h_{j}^{i}} h_{i l} h_{l j} \kappa_{\epsilon}$.

Note that if $\kappa:=\frac{G}{H}$, we have

$$
\frac{\partial \kappa}{\partial h_{p}^{q}} h_{m}^{q} h_{p}^{m}=\sum_{i=1}^{2} \frac{\partial \kappa}{\partial \lambda_{i}} \lambda_{i}^{2}=2 \kappa^{2}
$$

hence

$$
\frac{\partial \kappa_{\epsilon}}{\partial h_{p}^{q}} h_{m}^{q} h_{p}^{m}=\sum_{i=1}^{2} \frac{\partial \kappa_{\epsilon}}{\partial \lambda_{i}} \lambda_{i}^{2}=2 \kappa^{2}+\epsilon|A|^{2}
$$

with $|A|^{2}=\lambda_{1}^{2}+\lambda_{2}^{2}$. We then conclude from the above lemma that $H$ and $\kappa_{\epsilon}$ satisfy the evolution equations

$$
\frac{\partial}{\partial t} H=\mathcal{L}_{\epsilon} H+\frac{\partial^{2} \kappa_{\epsilon}}{\partial h_{q}^{p} \partial h_{m}^{l}} \nabla^{i} h_{q}^{p} \nabla_{j} h_{m}^{l}+\left(2 \kappa^{2}+\epsilon|A|^{2}\right) H
$$

and

$$
\frac{\partial \kappa_{\epsilon}^{2}}{\partial t}=\mathcal{L}_{\epsilon} \kappa_{\epsilon}+\left(2 \kappa^{2}+\epsilon|A|^{2}\right) \kappa_{\epsilon}^{2} .
$$

We will now combine the above evolution equations to establish the following uniform bound on the second fundamental form. 
Proposition 2.4. There exist uniform constants $C$ and $T_{0}$ so that

$$
\max _{\Sigma_{t}^{\epsilon}}|A| \leq C, \quad \forall t \in\left[0, \min \left(T_{\epsilon}, T_{0}\right)\right) .
$$

Proof. Recall that $H$ satisfies the equation (2.5). If we multiply this equation by $H$, we get

$$
\frac{\partial H^{2}}{\partial t}=\mathcal{L}_{\epsilon}\left(H^{2}\right)-2 a_{\epsilon}^{i k} \nabla_{i} H \nabla_{k} H+\frac{\partial^{2} \kappa_{\epsilon}}{\partial h_{q}^{p} \partial h_{m}^{l}} \nabla^{i} h_{q}^{p} \nabla_{i} h_{m}^{l} \cdot H+2\left(2 \kappa^{2}+\epsilon|A|^{2}\right) H^{2}
$$

with $\kappa=G / H$. Notice that the definiteness of the matrix $D^{2} \kappa_{\epsilon}=\left[\frac{\partial^{2} \kappa_{\epsilon}}{\partial h_{p}^{q} \partial h_{m}^{l}}\right]$ depends on the sign of $H$. It is easier to check this in geodesic coordinates around a point at which the Weingarten map is diagonalized. In those coordinates, by (1.2), we have

$$
\sum_{p, q, m, l} \frac{\partial^{2} \kappa_{\epsilon}}{\partial h_{q}^{p} \partial h_{m}^{l}} \nabla^{i} h_{q}^{p} \nabla_{i} h_{m}^{l}=\sum_{p, q} \frac{\partial^{2} \kappa_{\epsilon}}{\partial \lambda_{p} \lambda_{q}} \nabla^{i} h_{p}^{p} \nabla_{i} h_{q}^{q}+\sum_{p \neq q} \frac{\frac{\partial \kappa_{\epsilon}}{\partial \lambda_{p}}-\frac{\partial \kappa_{\epsilon}}{\partial \lambda_{q}}}{\lambda_{p}-\lambda_{q}}\left(\nabla_{i} h_{p}^{q}\right)^{2}
$$

where the matrix $D^{2} \kappa_{\epsilon}:=\left[\frac{\partial^{2} \kappa_{\epsilon}}{\partial \lambda_{p} \lambda_{q}}\right]$ is given by

$$
D^{2} \kappa_{\epsilon}=\left(\begin{array}{cc}
-\frac{2 \lambda_{2}^{2}}{\left(\lambda_{1}+\lambda_{2}\right)^{3}} & \frac{2 \lambda_{1} \lambda_{2}}{\left(\lambda_{1}+\lambda_{2}\right)^{3}} \\
\frac{2 \lambda_{1} \lambda_{2}}{\left(\lambda_{1}+\lambda_{2}\right)^{3}} & -\frac{2 \lambda_{1}^{2}}{\left(\lambda_{1}+\lambda_{2}\right)^{3}}
\end{array}\right)=-\frac{2}{H^{3}}\left(\begin{array}{cc}
\lambda_{2}^{2} & -\lambda_{1} \lambda_{2} \\
-\lambda_{1} \lambda_{2} & \lambda_{1}^{2}
\end{array}\right)
$$

and for $p \neq q$,

$$
\frac{\frac{\partial \kappa_{\epsilon}}{\partial \lambda_{p}}-\frac{\partial \kappa_{\epsilon}}{\partial \lambda_{q}}}{\lambda_{p}-\lambda_{q}}=\frac{\lambda_{q}^{2}-\lambda_{p}^{2}}{\lambda_{p}-\lambda_{q}}=-\frac{1}{H}
$$

It is now easy to see that

$$
\frac{\partial^{2} \kappa_{\epsilon}}{\partial h_{q}^{p} \partial h_{m}^{l}} \nabla^{i} h_{q}^{p} \nabla_{i} h_{m}^{l} \cdot H \leq 0
$$

hence

$$
\frac{\partial H^{2}}{\partial t} \leq \mathcal{L}_{\epsilon}\left(H^{2}\right)+2\left(2 \kappa^{2}+\epsilon|A|^{2}\right) H^{2} .
$$

Similarly, from the evolution of $\kappa_{\epsilon}$, namely (2.6), we obtain

$$
\frac{\partial \kappa_{\epsilon}^{2}}{\partial t} \leq \mathcal{L}_{\epsilon}\left(\kappa_{\epsilon}^{2}\right)+2\left(2 \kappa^{2}+\epsilon|A|^{2}\right) \kappa_{\epsilon}^{2} .
$$

We observe that because of the appearance of the second fundamental form $|A|^{2}$ in the zero order term of the equations (2.10) and (2.11), we cannot estimate the maximum of $H^{2}$ and $\kappa_{\epsilon}^{2}$ directly from each equation using the maximum principle. This is because the surface is not convex. However, it is possible to estimate the 
THE HARMONIC MEAN CURVATURE FLOW OF NONCONVEX SURFACES IN $\mathbb{R}^{3} \quad 7$ maximum of $H^{2}+\kappa_{\epsilon}^{2}$ by combining the two evolution equations. To this end, we set $M=H^{2}+\kappa_{\epsilon}^{2}$ and compute, by adding the last two equations, that

$$
\frac{\partial M}{\partial t} \leq \mathcal{L}_{\epsilon} M+2\left(2 \kappa^{2}+\epsilon|A|^{2}\right) M
$$

We will show the bound

$$
2 \kappa^{2}+\epsilon|A|^{2} \leq C\left(H^{2}+\kappa_{\epsilon}^{2}\right)
$$

for some uniform in $\epsilon$ constant $C$, where $\kappa_{\epsilon}=\kappa+\epsilon H$ and $\kappa=G / H$. Since $\kappa \leq \kappa_{\epsilon}$ it will be sufficient to show that

$$
|A|^{2} \leq C\left(H^{2}+\kappa^{2}\right)
$$

Expressing everything in terms of the principal curvatures $\lambda_{1}$ and $\lambda_{2}$, the above reduces to the estimate

$$
\lambda_{1}^{2}+\lambda_{2}^{2} \leq C\left(\left(\lambda_{1}+\lambda_{2}\right)^{2}+\left(\frac{\lambda_{1} \lambda_{2}}{\lambda_{1}+\lambda_{2}}\right)^{2}\right) .
$$

If $\lambda_{2}=0$ the above inequality is clearly satisfied. Assume that $\lambda_{2} \leq \lambda_{1}$ with $\lambda_{2} \neq 0$ and set $\mu=\lambda_{1} / \lambda_{2}$. Since $H=\lambda_{1}+\lambda_{2}>0$, we conclude that $|\mu| \geq 1$. Then, the last inequality is expressed as

$$
1+\frac{1}{\mu^{2}} \leq C\left(\frac{(1+\mu)^{2}}{\mu^{2}}+\frac{1}{(1+\mu)^{2}}\right)
$$

which can be reduced to showing that

$$
\frac{(1+\mu)^{2}}{\mu^{2}}+\frac{1}{(1+\mu)^{2}} \geq c>0
$$

for a uniform constant $c$, since $|\mu| \geq 1$. This inequality is clearly satisfied when $|\mu| \geq 1$. Hence, (2.13) holds.

Applying (2.13) on (2.12) we conclude get

$$
\frac{\partial M}{\partial t} \leq \mathcal{L}_{\epsilon} M+\theta M^{2}
$$

for some uniform constant $\theta$. The maximum principle then implies the differential inequality

$$
\frac{d M_{\max }}{d t} \leq \theta M_{\max }^{2}
$$

which readily implies that

$$
\max _{\Sigma_{t}^{\epsilon}} M \leq C, \quad \forall t \in\left[0, \min \left(T_{\epsilon}, T_{0}\right)\right)
$$

for some uniform in $\epsilon$ constants $C$ and $T_{0}$. This combined with (2.14) implies (2.7) finishing the proof of the proposition. 
To establish the short existence of the flow ( $\mathrm{HMCF}$ on $\left(0, T_{0}\right)$ for some $T_{0}>0$, we still need to bound $H$ from below away from zero independently of $\epsilon$. This does not follow naturally from the evolution of $H$, because the equation (2.5) carries a quadratic negative term which depends on the derivatives of the second fundamental form. Hence to establish the lower bound on $H$ we need to combine the evolution of $H$ with the evolution of the gradient of the second fundamental form. This is shown in the next proposition.

Proposition 2.5. There exist uniform in $\epsilon$ positive constants $T_{0}, C$ and $\delta$, so that

$$
|\nabla A| \leq C \quad \text { and } \quad H \geq \delta, \quad \text { on } \Sigma_{t}^{\epsilon}
$$

for $t \in\left[0, \min \left(T_{\epsilon}, T_{0}\right)\right)$.

Proof. We will first compute the evolution equation for $\sum_{i, j}\left|\nabla h_{i}^{j}\right|^{2}$. Lets first see how $h_{i}^{j}$ evolves. We have

$$
\frac{\partial}{\partial t} h_{i}^{j}=\mathcal{L}_{\epsilon}\left(h_{i}^{j}\right)+\frac{\partial^{2} \kappa_{\epsilon}}{\partial h_{q}^{p} \partial h_{m}^{l}} \nabla^{i} h_{q}^{p} \nabla^{j} h_{m}^{l}+\frac{\partial \kappa_{\epsilon}}{\partial h_{m}^{l}} h_{p}^{l} h_{m}^{p} h_{j}^{i} .
$$

¿From the previous equation, commuting derivatives we get

$$
\begin{aligned}
\frac{\partial}{\partial t} \nabla_{r} h_{i}^{j} & =\mathcal{L}_{\epsilon}\left(\nabla_{r} h_{i}^{j}\right)+\frac{\partial^{2} \kappa_{\epsilon}}{\partial h_{p}^{q} \partial h_{n}^{s}} \nabla_{r} h_{n}^{s} \nabla_{p} \nabla_{q} h_{i}^{j}+\frac{\partial \kappa_{\epsilon}}{\partial h_{p q}} R_{r p q m} \nabla_{m} h_{i}^{j} \\
& +\frac{\partial^{3} \kappa_{\epsilon}}{\partial h_{p}^{q} \partial h_{m}^{l} \partial h_{n}^{s}} \nabla_{r} h_{n}^{s} \nabla^{i} h_{p}^{q} \nabla_{j} h_{m}^{l}+\frac{\partial^{2} \kappa_{\epsilon}}{\partial h_{p} q \partial h_{m}^{l}} \nabla_{r} \nabla^{i} h_{p}^{q} \nabla_{j} h_{m}^{l}+ \\
& +\frac{\partial^{2} \kappa_{\epsilon}}{\partial h_{p} q \partial h_{m}^{l}} \nabla^{i} h_{p}^{q} \nabla_{r} \nabla_{j} h_{m}^{l}+\frac{\partial^{2} \kappa_{\epsilon}}{\partial h_{m}^{l} \partial h_{n}^{s}} \nabla_{r} h_{n}^{s} h_{p}^{l} h_{m}^{p} h_{j}^{i}+ \\
& +\frac{\partial \kappa_{\epsilon}}{\partial h_{m}^{l}} \nabla_{r} h_{p}^{l} h_{m}^{p} h_{i}^{j}+\frac{\partial \kappa_{\epsilon}}{\partial h_{m}^{l}} h_{p}^{l} \nabla_{r} h_{m}^{p} h_{i}^{j}+\frac{\partial \kappa_{\epsilon}}{\partial h_{m}^{l}} h_{p}^{l} h_{m}^{p} \nabla_{r} h_{i}^{j}
\end{aligned}
$$

Let $w=\sum_{i, j}\left|\nabla h_{i}^{j}\right|^{2}$. Since $\left|\nabla h_{i}^{j}\right|^{2}=g^{p q} \nabla_{p} h_{i}^{j} \nabla_{q} h_{i}^{j}$ and $\frac{\partial g_{i j}}{\partial t}=2 \kappa_{\epsilon} h_{i j}$, we get

$$
\begin{aligned}
\frac{\partial w}{\partial t} & =-4 g^{p a} g^{q b} \kappa_{\epsilon} h_{a b} \nabla_{p} h_{i}^{j} \nabla_{q} h_{i}^{j}+\mathcal{L}_{\epsilon}(w)-2 \dot{\kappa}_{\epsilon}\left(\nabla^{2} h_{i}^{j}, \nabla^{2} h_{i}^{j}\right)+ \\
& +g^{p q} \frac{\partial \kappa_{\epsilon}}{\partial h_{a}^{b}} R_{p a b s} \nabla_{s} h_{i}^{j} \nabla_{q} h_{i}^{j}+\frac{\partial^{2} \kappa_{\epsilon}}{\partial h_{p}^{q} \partial h_{m}^{l}} g^{r a} \nabla_{r} h_{m}^{l} \nabla_{p} \nabla_{q} h_{i}^{j} \nabla_{a} h_{i}^{j}+ \\
& +\frac{\partial^{3}}{\partial h_{p}^{q} \partial h_{m}^{l} \partial h_{n}^{s}} g^{r a} \nabla_{r} h_{n}^{s} \nabla^{i} h_{p}^{q} \nabla_{j} h_{m}^{l} \nabla_{a} h_{i}^{j}+\frac{\partial^{2} \kappa_{\epsilon}}{\partial h_{p}^{q} \partial h_{m}^{l}} g^{r a} \nabla_{r} \nabla^{i} h_{p}^{q} \nabla_{j} h_{m}^{l} \nabla_{a} h_{i}^{j}+ \\
& +\frac{\partial^{2} \kappa_{\epsilon}}{\partial h_{p}^{q} \partial h_{m}^{l}} g^{r a} \nabla^{i} h_{p}^{q} \nabla_{r} \nabla_{j} h_{m}^{l} \nabla_{a} h_{i}^{j}+\frac{\partial^{2} \kappa_{\epsilon}}{\partial h_{m}^{l} \partial h_{n}^{s}} g^{r a} \nabla_{r} h_{n}^{s} h_{p}^{l} h_{m}^{p} h_{i}^{j}+ \\
& +\frac{\partial \kappa_{\epsilon}}{\partial h_{m}^{l}} g^{r a} \nabla_{r} h_{p}^{l} h_{m}^{p} h_{i}^{j} \nabla_{a} h_{i}^{j}+\frac{\partial \kappa_{\epsilon}}{\partial h_{m}^{l}} g^{r a} h_{p}^{l} \nabla_{r} h_{m}^{p} h_{i}^{j} \nabla_{a} h_{i}^{j}+ \\
& +\frac{\partial \kappa_{\epsilon}}{\partial h_{m}^{l}} g^{r a} h_{p}^{l} h_{m}^{p} \nabla_{r} h_{i}^{j} \nabla_{a} h_{i}^{j} .
\end{aligned}
$$


Whenever we see $i$ and $j$ in the previous equation we assume that we are summing over all indices $i$ and $j$. Also,

$$
\dot{\kappa}_{\epsilon}\left(\nabla^{2} h_{i}^{j}, \nabla^{2} h_{i}^{j}\right)=\frac{\partial \kappa_{\epsilon}}{\partial h_{p}^{q}} g^{p q} g^{c d} \nabla_{q} \nabla_{c} h_{i}^{j} \nabla_{p} \nabla_{d} h_{i}^{j} .
$$

Notice that since $|A| \leq C$ for all $t \in\left[0, \min \left(T_{\epsilon}, T_{0}\right)\right)$, we have

$$
\left|\frac{\partial^{2} \kappa_{\epsilon}}{\partial h_{p}^{q} \partial h_{m}^{l}}\right| \leq \frac{C_{1}}{H^{3}} \quad \text { and } \quad\left|\frac{\partial^{3} \kappa_{\epsilon}}{\partial h_{p}^{q} \partial h_{m}^{l} \partial h_{n}^{s}}\right| \leq \frac{C_{1}}{H^{4}}
$$

for a uniform constant $C$.

We next compute the evolution equation for $1 / H$ from the evolution of $H$, namely equation (2.5). By direct computation we get that

$$
\frac{\partial}{\partial t}\left(\frac{1}{H}\right)=\mathcal{L}_{\epsilon}\left(\frac{1}{H}\right)-\frac{2}{H^{3}} \frac{\partial \kappa_{\epsilon}}{\partial h_{p}^{q}} \nabla_{p} H \nabla_{q} H-\frac{1}{H^{2}} \frac{\partial^{2} \kappa_{\epsilon}}{\partial h_{p}^{q} \partial h_{m}^{l}} \nabla^{i} h_{p}^{q} \nabla_{i} h_{m}^{l}-\frac{1}{H} \frac{\partial \kappa_{\epsilon}}{\partial h_{m}^{l}} h_{p}^{l} h_{m}^{p} .
$$

Taking away the second negative term on the right hand side we easily conclude the differential inequality

$$
\frac{\partial}{\partial t}\left(\frac{1}{H}\right) \leq \mathcal{L}_{\epsilon}\left(\frac{1}{H}\right)+\frac{C w}{H^{5}}+\frac{C}{H} .
$$

Combining the evolution equations of $w$ and $1 / H$ we will now compute the evolution equation for

$$
\mathcal{V}:=w+\frac{1}{H}
$$

We look at the point $(P, t)$ at which $\mathcal{V}$ achieves its maximum at time $t$ and choose coordinates around $P$ so that both, the second fundamental form and the metric matrix are diagonal at $P$. Using the exact form of coefficients $a_{\epsilon}^{i k}=\frac{\partial \kappa_{\epsilon}}{\partial h_{p}^{q}}$ computed in (2.1) we get

$$
\begin{aligned}
-2 \sum_{i, j} \dot{\kappa}_{\epsilon}\left(\nabla^{2} h_{i}^{j}, \nabla^{2} h_{i}^{j}\right)= & -2 \sum_{i, j} \frac{\partial \kappa_{\epsilon}}{\partial h_{p}^{q}} g^{c d} \nabla_{q} \nabla_{c} h_{i}^{j} \nabla_{p} \nabla_{d} h_{i}^{j} \\
= & -\frac{2}{H^{2}} \sum_{i, j}\left[\lambda_{2}^{2}\left(\nabla_{1} \nabla_{1} h_{i}^{j}\right)^{2}+\lambda_{1}^{2}\left(\nabla_{2} \nabla_{2} h_{i}^{j}\right)^{2}\right. \\
& \left.+\left(\lambda_{1}^{2}+\lambda_{2}^{2}\right)\left(\nabla_{1} \nabla_{2} h_{i}^{j}\right)^{2}\right] .
\end{aligned}
$$

Our goal is to absorb all the remaining terms that contain the second order derivatives, appearing in the evolution equation for $\mathcal{V}$, in the good term (2.16). By looking at the evolution equation of $w$, we see that those second order terms are

$$
\begin{gathered}
\mathcal{O}=\frac{\partial^{2} \kappa_{\epsilon}}{\partial h_{p}^{q} \partial h_{m}^{l}} g^{r a} \nabla_{r} h_{m}^{l} \nabla_{p} \nabla_{q} h_{i}^{j} \nabla_{a} h_{i}^{j}, \\
\mathcal{P}=\frac{\partial^{2} \kappa_{\epsilon}}{\partial h_{p}^{q} \partial h_{m}^{l}} g^{r a} \nabla_{r} \nabla^{i} h_{p}^{q} \nabla_{j} h_{m}^{l} \nabla_{a} h_{i}^{j}
\end{gathered}
$$


and

$$
\mathcal{R}=\frac{\partial^{2} \kappa_{\epsilon}}{\partial h_{p}^{q} \partial h_{m}^{l}} g^{r a} \nabla^{i} h_{p}^{q} \nabla_{r} \nabla_{j} h_{m}^{l} \nabla_{a} h_{i}^{j}
$$

where we understand summing over all indices. Denote by $\xi_{m}^{l}:=\nabla_{r} h_{m}^{l}$ and by $\eta_{p}^{q}:=\nabla_{p} \nabla_{q} h_{i}^{j}$. If we specify the coordinates around the maximum point $P$ in which $W$ and $g$ are diagonal, by (1.2)

$$
\begin{aligned}
\mathcal{O} & =g^{r a} \nabla_{r} h_{i}^{j}\left(\sum_{p, q} \frac{\partial^{2} \kappa}{\partial \lambda_{p} \lambda_{q}} \xi_{p}^{p} \eta_{q}^{q}+\sum_{p \neq q} \frac{\frac{\partial \kappa}{\partial \lambda_{p}}-\frac{\partial \kappa}{\partial \lambda_{q}}}{\lambda_{p}-\lambda_{q}} \xi_{p}^{q} \eta_{p}^{q}\right) \\
& =\nabla_{r} h_{i}^{j}\left(-\frac{2 \lambda_{2}^{2}}{H^{3}} \nabla_{r} h_{1}^{1} \nabla_{1} \nabla_{1} h_{i}^{j}-\frac{2 \lambda_{1}^{2}}{H^{3}} \nabla_{r} h_{2}^{2} \nabla_{2} \nabla_{2} h_{i}^{j}+\frac{2 \lambda_{1} \lambda_{2}}{H^{3}} \nabla_{r} h_{1}^{1} \nabla_{2} \nabla_{2} h_{i}^{j}+\right. \\
& +\frac{2 \lambda_{1} \lambda_{2}}{H^{3}} \nabla_{r} h_{2}^{2} \nabla_{1} \nabla_{1} h_{i}^{j}-\frac{1}{H}\left(\nabla_{1} \nabla_{2} h_{i}^{j} \nabla_{r} h_{1}^{2}+\nabla_{2} \nabla_{1} h_{i}^{j} \nabla_{r} h_{2}^{1}\right)
\end{aligned}
$$

Since $|A| \leq C$ and

$$
\frac{1}{H}=\frac{\lambda_{1}+\lambda_{2}}{H^{2}} \leq \frac{\left|\lambda_{1}\right|+\left|\lambda_{2}\right|}{H^{2}}
$$

by the Cauchy-Schwartz inequality, we can estimate $\mathcal{O}$ term by term, namely

$$
\begin{aligned}
\left|2 \nabla_{r} h_{i}^{j} \frac{\lambda_{2}^{2}}{H^{3}} \nabla_{r} h_{1}^{1} \nabla_{1} \nabla_{1} h_{i}^{j}\right| & =\left|2 \nabla_{r} h_{i}^{j} \frac{\lambda_{2}}{H^{2}} \nabla_{r} h_{1}^{1}\right|\left|\frac{\lambda_{2}}{H} \nabla_{1} \nabla_{1} h_{i}^{j}\right| \\
& \leq C \frac{w^{2}}{H^{4}}+\beta_{1} \frac{\lambda_{2}^{2}}{H^{2}}\left|\nabla_{1} \nabla_{1} h_{i}^{j}\right|^{2} \\
& \leq C \frac{w^{2}}{H^{4}}+\beta_{1} \sum_{i j} \dot{\kappa}_{\epsilon}\left(\nabla^{2} h_{i}^{j} \nabla^{2} h_{i}^{j}\right)
\end{aligned}
$$

and

$$
\begin{aligned}
\left|2 \frac{\lambda_{1} \lambda_{2}}{H^{3}} \nabla_{r} h_{i}^{j} \nabla_{r} h_{1}^{1} \nabla_{1} \nabla_{1} h_{i}^{j}\right| & =\left|2 \frac{\lambda_{1}}{H^{2}} \nabla_{r} h_{1}^{1} \nabla_{r} h_{i}^{j}\right|\left|\frac{\lambda_{2}}{H} \nabla_{1} \nabla_{i} h_{i}^{j}\right| \\
& \leq C \frac{w^{2}}{H^{4}}+\beta_{1} \frac{\lambda_{2}^{2}}{H^{2}}\left|\nabla_{1} \nabla_{1} h_{i}^{j}\right| \\
& \leq C \frac{w^{2}}{H^{4}}+\beta_{1} \sum_{i j} \dot{\kappa}_{\epsilon}\left(\nabla^{2} h_{i}^{j} \nabla^{2} h_{i}^{j}\right)
\end{aligned}
$$

and

$$
\begin{aligned}
\left|\frac{1}{H} \nabla_{1} \nabla_{2} h_{i}^{j} \nabla_{r} h_{1}^{2} \nabla_{r} h_{i}^{j}\right| & \leq\left|\frac{1}{H^{2}} \nabla_{r} h_{1}^{2} \nabla_{r} h_{i}^{j}\right|\left|\frac{\lambda_{1}+\lambda_{2}}{H} \nabla_{1} \nabla_{2} h_{i}^{j}\right| \\
& \leq C \frac{w^{2}}{H^{4}}+\beta_{1}\left(\lambda_{1}^{2}+\lambda_{2}^{2}\right)\left|\nabla_{1} \nabla_{2} h_{i}^{j}\right|^{2} \\
& \leq C \frac{w^{2}}{H^{4}}+\beta_{1} \sum_{i j} \dot{\kappa}_{\epsilon}\left(\nabla^{2} h_{i}^{j}, \nabla^{2} h_{i}^{j}\right)
\end{aligned}
$$


THE HARMONIC MEAN CURVATURE FLOW OF NONCONVEX SURFACES IN $\mathbb{R}^{3} \quad 11$

where $\beta_{1}>0$ is a uniform small number. We can estimate other terms in $\mathcal{O}$ the same way and combining all those estimates yield

$$
|\mathcal{O}| \leq C \frac{w^{2}}{H^{4}}+\beta \sum_{i, j} \dot{\kappa}_{\epsilon}\left(\nabla^{2} h_{i}^{j}, \nabla^{2} h_{i}^{j}\right)
$$

where $\beta>0$ is a small fixed number.

In order to estimate $\mathcal{P}$, we would like to be able somehow to switch the pair of indices $\{i, r\}$ with $\{p, q\}$ so that we reduce estimating $\mathcal{P}$ to the previous case of $\mathcal{O}$. We will use Gauss-Codazzi equations in the form

$$
\nabla_{l} h_{i j}=\nabla_{i} h_{l j} .
$$

In our special coordinates at the point we have

$$
\begin{aligned}
\nabla_{r} \nabla^{i} h_{p}^{q} & =\nabla_{r} \nabla^{i}\left(h_{p s} g^{q s}\right)=\nabla_{r}\left(g^{i j} \nabla_{j}\left(h_{p s} g^{q s}\right)\right) \\
& =\nabla_{r}\left(g^{i j} g^{q s}\right) \cdot \nabla_{j} h_{p s}+g^{i j} g^{q s} \nabla_{r} \nabla_{j} h_{p s}+h_{p s} \nabla_{r}\left(g^{i j} \nabla_{j} g^{q s}\right) \\
& =\nabla_{p} \nabla_{q} h_{r j}+\nabla_{r}\left(g^{i j} g^{q s}\right) \cdot \nabla_{j} h_{p s}+h_{p p} \nabla_{r}\left(g^{i j} \nabla_{j} g^{p q}\right)
\end{aligned}
$$

We have the following:

Claim. There is a uniform constant $\tilde{C}$ depending on $C$, so that

$$
|g(\cdot, t)|_{C^{2}} \leq \tilde{C}
$$

as long as $|A| \leq C$.

To prove (2.19) we observe that in geodesic coordinates $\left\{x_{i}\right\}$ around a point $p$, which corresponds to the origin in geodesic coordinates, we have

$$
g_{i j}(x)=\delta_{i j}+\frac{1}{3} R_{i p q j} x^{p} x^{q}+O\left(|x|^{3}\right)
$$

and that an easy computation shows that

$$
\nabla_{p} \nabla_{q} g_{i j}(0)=-\frac{1}{3} R_{i p q j} .
$$

By the Gauss equations, we have $R_{i p q j}=h_{i q} h_{p j}-h_{i j} h_{p q}$, which yields to $\left|\nabla_{p} \nabla_{q} g_{i j}\right| \leq$ $\tilde{C}$ as long as $|A| \leq C$. This together with (2.20) proves the Claim.

Combining (2.18) - (2.19), we obtain as in the estimate of $\mathcal{O}$, the bound

$$
|\mathcal{P}| \leq \frac{C w^{2}}{H^{4}}+\beta \sum_{i, j} \dot{\kappa}_{\epsilon}\left(\nabla^{2} h_{i}^{j}, \nabla^{2} h_{i}^{j}\right) .
$$

Similarly, we get the estimate for $\mathcal{R}$. We conclude that

$$
|\mathcal{O}|+|\mathcal{P}|+|\mathcal{R}| \leq \frac{C w^{2}}{H^{4}}+3 \beta \sum_{i, j} \dot{\kappa}_{\epsilon}\left(\nabla^{2} h_{i}^{j}, \nabla^{2} h_{i}^{j}\right) .
$$


Choosing $\beta>0$ so that $3 \beta<2$ in (2.21) and analyzing the right hand side of the evolution of $w$ term by term, we obtain the following estimate at the maximum point $P$ of $\mathcal{V}$ at time $t$

$$
\frac{d \mathcal{V}_{\max }}{d t} \leq C w+\frac{C w^{2}}{H^{4}}+\frac{C \sqrt{w}}{H^{3}}+\frac{C w}{H^{5}}+\frac{C}{H} .
$$

Young's inequlity, implies the estimates

$$
\frac{w^{2}}{H^{4}} \leq w^{6}+\frac{1}{H^{6}} \leq \mathcal{V}^{6}
$$

and

$$
\frac{w}{H^{5}} \leq w^{6}+\frac{1}{H^{6}} \leq \mathcal{V}^{6}
$$

and

$$
\frac{\sqrt{w}}{H^{3}} \leq w+\frac{1}{H^{6}} \leq \mathcal{V}+\mathcal{V}^{6}
$$

Hence, denoting by $f(t)=\mathcal{V}_{\max }(t)$ we obtain

$$
\frac{d f}{d t} \leq C\left(f+f^{6}\right)
$$

which implies the existence of uniform constants $\bar{C}$ and $T_{0}$, depending only on $C$ and $f(0)$, so that

$$
\sup _{\Sigma_{t}^{\epsilon}}\left(\frac{1}{H}+\sum_{i, j}\left|\nabla h_{i}^{j}\right|^{2}\right) \leq \bar{C}, \quad \text { for all } t \in\left[0, \min \left(T_{\epsilon}, T_{0}\right)\right) .
$$

This finishes the proof of the lemma.

Having all the curvature estimates (that are proved above), we can justify the short time existence of the $C^{2,1}$-solution to the (HMCF).

Proof of Theorem [2.1. For every $\epsilon>0$, let $T_{\epsilon}$ be the maximal time so that

$$
|A|_{C^{1}\left(\Sigma_{t}^{\epsilon}\right)} \leq C, \quad \text { and } \quad H \geq \delta>0
$$

where $C, \delta$ are constants taken from Proposition 2.5. Take now $\epsilon_{i} \rightarrow 0$. We have that $|A|_{C^{1}\left(\Sigma_{t}^{\epsilon_{i}}\right)} \leq C$, which implies $\left|F_{\epsilon_{i}}\right|_{C^{2,1}} \leq C$, for all $t \in\left[0, T_{0}\right]$. By the ArzelaAscoli theorem there is a subsequence so that $F_{\epsilon_{i}}(\cdot, t) \stackrel{C^{2,1}}{\rightarrow} F(\cdot, t)$, where $F(\cdot, t)$ is a $C^{2,1}$ solution to ( $(\mathrm{HMCF})$. Since we have a comparison principle for $C^{2,1}$ solutions to (1.1) as discussed above, the uniqueness of a $C^{2,1}$ solution immediately follows. 


\section{LONG TIME EXISTENCE FOR THE $\epsilon$-FLOW}

In this section we will study the long time existence for the $\epsilon$-regularized flow $\left(\mathrm{HMCF}_{\epsilon}\right)$ assuming that $\Sigma_{0}$ is an arbitrary smooth surface with mean curvature $H>0$, Euler characteristic $\chi\left(\Sigma_{0}\right)>0$ and it is star-shaped with respect to the origin. Throughout the section we fix $\epsilon>0$ sufficiently small, we denote by $\Sigma_{t}^{\epsilon}$ the surface evolving by $\left(\mathrm{HMCF}_{\epsilon}\right)$ and, to simplify the notation, we drop the index $\epsilon$ from $F, \nu, H, G, \kappa, A, g_{i j}, h_{i j}$ etc. The $\epsilon$-flow has one obvious advantage over (1.1), it is not degenerate and therefore it has smoothing properties. Indeed, it follows from the Krylov and Schauder estimates that a $C^{1,1}$ solution of $\left(\mathrm{HMCF}_{\epsilon}\right)$ is $C^{\infty}$ smooth.

Assume that $\Sigma_{t}^{\epsilon}$ is a solution of $\left(\mathrm{HMCF}_{\epsilon}\right)$ on $\left[0, T_{\epsilon}\right)$ and let us consider the evolution equation for the area form $d \mu_{t}$, namely

$$
\frac{\partial}{\partial t} d \mu_{t}=-2\left(\frac{G}{H}+\epsilon H\right) \frac{H}{2} d \mu_{t}=-\left(G+\epsilon H^{2}\right) d \mu_{t} .
$$

Integrating it over the surface $\Sigma_{t}^{\epsilon}$ we obtain the following ODE for the total area $\mu_{t}\left(\Sigma_{t}^{\epsilon}\right)$ of the surface $\Sigma_{t}^{\epsilon}$

$$
\frac{d}{d t} \mu_{t}\left(\Sigma_{t}^{\epsilon}\right)=-\int_{\Sigma_{t}^{\epsilon}}\left(G+\epsilon H^{2}\right) d \mu_{t}
$$

By the Gauss-Bonnet formula we have

$$
\int_{\Sigma_{t}^{\epsilon}} G d \mu_{t}=2 \pi \chi\left(\Sigma_{t}\right)
$$

Since $\Sigma_{0}$ is a surface with positive Euler characteristic, then by the uniformization theorem $\chi\left(\Sigma_{t}\right)=2$ and therefore we conclude the equation

$$
\frac{d}{d t} \mu_{t}\left(\Sigma_{t}^{\epsilon}\right)=-4 \pi-\epsilon \int_{\Sigma_{t}^{\epsilon}} H^{2} d \mu_{t}
$$

Denote by $T_{\epsilon}$ the maximum time of existence of $\left(\mathrm{HMCF}_{\epsilon}\right)$. Integrating (3.1) in time from 0 to $T_{\epsilon}$, solving with respect of $T_{\epsilon}$ and using that $\mu_{t}\left(\Sigma_{t}^{\epsilon}\right) \geq 0$, gives

$$
T_{\epsilon} \leq \frac{1}{4 \pi} \mu_{0}\left(\Sigma_{0}\right)-\frac{\epsilon}{4 \pi} \int_{0}^{T_{\epsilon}} \int_{\Sigma_{t}^{\epsilon}} H^{2} d \mu_{t}
$$

This, in particular shows that

$$
T_{\epsilon} \leq \frac{1}{4 \pi} \mu_{0}\left(\Sigma_{0}\right)
$$

where $\mu_{0}\left(\Sigma_{0}\right)$ is the area of the initial surface $\Sigma_{0}$. 
Our goal is to prove the following result, concerning the long time existence of the flow $\left(\mathrm{HMCF}_{\epsilon}\right)$. We will also establish curvature bounds and curvature pinching estimates which are independent of $\epsilon$.

Theorem 3.1. Let $\Sigma_{0}$ be a compact star-shaped hyper-surface in $\mathbb{R}^{3}$ which is of class $C^{1,1}$ and has strictly positive mean curvature $H>0$. Then, there exists a maximal time of existence $T_{\epsilon}$ of a smooth $\left(\underline{\left.\mathrm{HMCF}_{\epsilon}\right)}\right.$ flow $\Sigma_{\epsilon}^{t}$ such that either:

(i) $H\left(P_{t}, t\right) \rightarrow 0$, as $t \rightarrow T_{\epsilon}$ at some points $P_{t} \in \Sigma_{t}^{\epsilon}$, or

(ii) $\Sigma_{\epsilon}^{t}$ shrinks to a point as $t \rightarrow T_{\epsilon}$ and $T_{\epsilon}$ is given explicitly by

$$
T_{\epsilon}=\frac{1}{4 \pi} \mu_{0}\left(\Sigma_{0}\right)-\frac{\epsilon}{4 \pi} \int_{0}^{T_{\epsilon}} \int_{\Sigma_{t}^{\epsilon}} H^{2} \mu_{t}
$$

where $\mu_{0}\left(\Sigma_{0}\right)$ is the total area of $\Sigma_{0}$. Moreover, $\int_{\Sigma_{t}^{\epsilon}} H^{2} d \mu_{t}$ is uniformly bounded for all $t \in\left[0, T_{\epsilon}\right)$, independently of $\epsilon$.

Assume that (i) does not happen in Theorem 3.1. Then, we have

$$
\min _{\Sigma_{\epsilon}^{t}} H(\cdot, t) \geq \delta>0, \text { for all } t \in\left[0, T_{\epsilon}\right)
$$

where $T_{\epsilon}$ is the maximal existence time of a smooth flow $\Sigma_{\epsilon}^{t}$.

Proposition 3.2. Assuming that (i) doesn't happen in Theorem [3.1, then the maximal time of existence $T$ of the flow $\left(\mathrm{HMCF}_{\epsilon}\right)$ satisfies $T \leq \mu_{0}\left(\Sigma_{0}\right) / 4 \pi$ and

$$
\limsup _{t \rightarrow T}|A|=\infty
$$

Proof. The bound $T \leq M_{0} / 4 \pi$ is proven above. Assume that $\max _{\Sigma_{\epsilon}^{t}}|A| \leq C$ for all $t \in[0, T)$. Then we want to show that the surfaces $\Sigma_{t}^{\epsilon}$ converge, as $t \rightarrow T$, to a smooth limiting surface $\Sigma_{T}^{\epsilon}$. Similarly as in [8], using the curvature bounds we have, for all $0<t_{t}<t_{2}<T$, the bounds

$$
\left|F\left(p, t_{1}\right)-F\left(p, t_{2}\right)\right| \leq C\left|t_{2}-t_{1}\right| \quad \text { and } \quad\left|\frac{\partial}{\partial t} g_{i j}\right|^{2} \leq C
$$

for a uniform in $t$ constant $C$, which imply that $F(\cdot, t)$ converges, as $t \rightarrow T$ to some continuous surface $\tilde{\Sigma}_{\epsilon}^{T}$. We get uniform $C^{2}$-bounds on $F$ out of the bound on $|A|$. Since our equation is uniformly parabolic and the operator $\kappa$ is concave, by Krylov and Schauder estimates we obtain all higher derivative bounds.

We have just shown that the surface $\Sigma_{T}^{\epsilon}$ is $C^{\infty}$ smooth. Also from our assumption

$$
H(\cdot, T) \geq \delta>0, \quad \text { on } \Sigma_{T}^{\epsilon}
$$


By Proposition 2.2 there exists $\tau_{\epsilon}>0$ for which a smooth flow can be continued on $\left[T, T+\tau_{\epsilon}\right)$, which contradicts our assumption that $T$ is maximal. Hence, $\lim \sup _{t \rightarrow T_{\epsilon}}|A|=\infty$ and the result follows.

3.1. Monotonicity formula. We will now show the monotonicity property of the quantity

$$
Q_{\epsilon}=\left\langle F_{\epsilon}, \nu\right\rangle+2 t \kappa_{\epsilon}
$$

along the flow $\left(\mathrm{HMCF}_{\epsilon}\right)$. This will play an essential role in establishing the long time existence. Similar quantity was considered by Smoczyk in [16].

Lemma 3.3. Assuming that $q_{\epsilon}(0):=\min _{\Sigma_{0}}\left\langle F_{\epsilon}, \nu\right\rangle \geq 0$, the quantity

$$
q_{\epsilon}(t):=\min _{\Sigma_{t}^{\epsilon}}\left(\left\langle F_{\epsilon}, \nu\right\rangle+2 t \kappa_{\epsilon}\right)
$$

is increasing in time for as long as the solution $\Sigma_{t}^{\epsilon}$ exists. Hence,

$$
q_{\epsilon}(t):=\min _{\Sigma_{t}^{\epsilon}}\left(\left\langle F_{\epsilon}, \nu\right\rangle+2 t \kappa_{\epsilon}\right) \geq q_{\epsilon}(0) \geq 0 .
$$

Proof. We will compute the evolution of $Q_{\epsilon}$ and apply the maximum principle. We begin by computing the evolution of $\left\langle F_{\epsilon}, \nu\right\rangle$. We have:

$$
\mathcal{L}_{\epsilon}\left(\left\langle F_{\epsilon}, \nu\right\rangle\right)=a_{\epsilon}^{i k} \nabla_{i}\left(\nabla_{k}\left\langle F_{\epsilon}, \nu\right\rangle\right)=a_{\epsilon}^{i k} \nabla_{i}\left(\left\langle e_{k}, \nu\right\rangle+\left\langle F_{\epsilon}, h_{k j} e_{j}\right\rangle\right),
$$

since

$$
\nabla_{i} \nu=h_{i j} e_{j}, \quad \nabla_{i} e_{j}=-h_{i j} \nu, \quad \nabla_{i} F_{\epsilon}=e_{i} .
$$

Using Gauss-Codazzi equation $\nabla_{i} h_{k j}=\nabla_{j} h_{i k}$ and since $\nabla_{i}\left(\left\langle e_{k}, \nu\right\rangle\right)=0$ we get

$$
\begin{aligned}
\mathcal{L}_{\epsilon}\left(\left\langle F_{\epsilon}, \nu\right\rangle\right) & =a_{\epsilon}^{i k}\left[\left\langle\nabla_{i} F_{\epsilon}, h_{k j} e_{j}\right\rangle+\left\langle F, h_{k j} \nabla_{i} e_{j}\right\rangle+\left\langle F, e_{j} \cdot \nabla_{i} h_{j k}\right\rangle\right] \\
& =a_{\epsilon}^{i k}\left[h_{i k}-h_{i j} h_{j k}\left\langle F_{\epsilon}, \nu\right\rangle+\left\langle F, e_{j} \cdot \nabla_{j} h_{i k}\right\rangle\right] \\
& =\kappa_{\epsilon}-a^{i k} h_{i j} h_{j k}\left\langle F_{\epsilon}, \nu\right\rangle+\left\langle F_{\epsilon}, \nabla \kappa_{\epsilon}\right\rangle .
\end{aligned}
$$

On the other hand, since $\frac{\partial \nu}{\partial t}=\nabla \kappa_{\epsilon}$, it follows

$$
\frac{\partial}{\partial t}\left\langle F_{\epsilon}, \nu\right\rangle=-\kappa_{\epsilon}+\left\langle F_{\epsilon}, \nabla \kappa_{\epsilon}\right\rangle
$$

which yields

$$
\frac{\partial}{\partial t}\left\langle F_{\epsilon}, \nu\right\rangle-\mathcal{L}_{\epsilon}\left(\left\langle F_{\epsilon}, \nu\right\rangle\right)=-2 \kappa_{\epsilon}+a^{i k} h_{i j} h_{j k}\left\langle F_{\epsilon}, \nu\right\rangle .
$$

We also have

$$
\frac{\partial \kappa_{\epsilon}}{\partial t}=\mathcal{L}_{\epsilon}\left(\kappa_{\epsilon}\right)+a_{\epsilon}^{i k} h_{i j} h_{j k} \kappa_{\epsilon} .
$$


Hence $Q_{\epsilon}=\left\langle F_{\epsilon}, \nu\right\rangle+2 t \kappa_{\epsilon}$ satisfies

$$
\frac{\partial Q_{\epsilon}}{\partial t}=\mathcal{L}_{\epsilon} Q_{\epsilon}+a_{\epsilon}^{i k} \nabla_{i} \nabla_{k} Q_{\epsilon}+a_{\epsilon}^{i k} h_{i j} h_{j k} Q_{\epsilon} .
$$

Notice that the right hand side of (3.5) is a strictly elliptic operator and

$$
a_{\epsilon}^{i k} h_{i j} h_{j k} \geq 0 .
$$

We conclude by the maximum principle that

$$
\left.q_{\epsilon}^{\prime}(t)=\frac{d}{d t}\left(\left\langle F_{\epsilon}, \nu\right\rangle+2 t \kappa_{\epsilon}\right\rangle\right)_{\min } \geq 0
$$

assuming that $q_{\epsilon}(0) \geq 0$. This implies that $q_{\epsilon}(t) \geq q_{\epsilon}(0)$ finishing the proof of the lemma.

Notice that if instead of $Q_{\epsilon}$ we take the quantity

$$
Q_{\eta, \epsilon}=\left\langle F_{\epsilon}, \nu\right\rangle+2(t+\eta) \kappa_{\epsilon}
$$

for any constant $\eta \in \mathbb{R}$, the same computation as above yields to that $Q_{\eta, \epsilon}$ satisfies

$$
\frac{\partial Q_{\eta, \epsilon}}{\partial t}=\mathcal{L}_{\epsilon} Q_{\eta, \epsilon}+a_{\epsilon}^{i k} \nabla_{i} \nabla_{k} Q_{\eta, \epsilon}+a_{\epsilon}^{i k} h_{i j} h_{j k} Q_{\eta, \epsilon} .
$$

Assume that at time $t=0$, we have

$$
q_{\eta, \epsilon}(0)=\min _{\Sigma_{0}}\left(\langle F, \nu\rangle+2 \eta \kappa_{\epsilon}\right) \geq 0
$$

for some $\eta \in \mathbb{R}$ (notice that $F_{\epsilon}=F$ at $t=0$ ). Then, the maximum principle to the above equation, gives:

Proposition 3.4. For any $\eta \in \mathbb{R}$, such that $q_{\eta, \epsilon}(0):=\min _{\Sigma_{0}}\left(\langle F, \nu\rangle+2 \eta \kappa_{\epsilon}\right) \geq 0$ the quantity

$$
q_{\eta, \epsilon}(t):=\min _{\Sigma_{t}^{\epsilon}}\left(\left\langle F_{\epsilon}, \nu\right\rangle+2(t+\eta) \kappa_{\epsilon}\right)
$$

is increasing in time. Hence

$$
q_{\epsilon, \eta}(t):=\min _{\Sigma_{t}^{\epsilon}}\left(\left\langle F_{\epsilon}, \nu\right\rangle+2(t+\eta) \kappa_{\epsilon}\right) \geq q_{\epsilon, \eta}(0) \geq 0
$$

Since the initial surface $\Sigma_{0}$ is star-shaped, we may choose $\eta>0$ so that we have $q_{\eta, \epsilon}(0)>0$. This is possible by continuity, since $\langle F, \nu\rangle>0$. By Proposition 3.4 we have

$$
Q_{\eta, \epsilon}(\cdot, t) \geq q_{\eta, \epsilon}(t)>0
$$

which implies the lower bound

$$
\kappa_{\epsilon}=\frac{G}{H}+\epsilon H \geq-\frac{\left\langle F_{\epsilon}, \nu\right\rangle}{2(t+\eta)} .
$$


We will show next that (3.7) implies a uniform lower bound on $\kappa_{\epsilon}$, independently of $\epsilon$. To this end, we need to bound $\left\langle F_{\epsilon}, \nu\right\rangle$, independently of $\epsilon$. This bound follows from the comparison principle for curvature flows with the property that the speed is an increasing function of the principal curvatures. More precisely, we have the following lemma.

Lemma 3.5. There exists a constant $C$, independent of $\epsilon$, so that

$$
\kappa_{\epsilon}:=\frac{G}{H}+\epsilon H \geq-C, \quad \forall t \in\left[0, T_{\epsilon}\right) .
$$

Proof. We claim that there is a uniform constant $C$ so that $\left|F_{\epsilon}\right| \leq C$, for all $t \in\left[0, T_{\epsilon}\right)$. To see that, let $\psi_{0}: S^{2} \rightarrow \mathbb{R}^{3}$ denote the parametrization of a sphere that encloses the initial hypersurface $\Sigma_{0}$. By the result of B. Andrews in [1], the solution $\psi_{\epsilon}(\cdot, t)$ of $\left(\overline{\mathrm{HMCF}}_{\epsilon}\right)$ with initial condition $\psi_{0}$ shrinks to a point in some finite time $\tilde{T}_{\epsilon}$. Moreover,

$$
\tilde{T}_{\epsilon} \leq \tilde{T}<\infty
$$

for a uniform constant $\tilde{T}$.

The standard comparison principle shows that the images of $F_{\epsilon}$ and $\psi_{\epsilon}$ stay disjoint for all the time of their existence. To see this, we consider the evolution of

$$
d(p, q, t):=\left|F_{\epsilon}(p, t)-\psi_{\epsilon}(q, t)\right|, \quad(p, q) \in \Sigma_{t}^{\epsilon} \times S^{2} .
$$

Assume that the minimum of $d$ at time $t$ occurs at $\left(p_{0}, q_{0}\right)$. If $W$ denotes the Weingarten map, at that minimum point $W\left(p_{0}\right) \geq W\left(q_{0}\right)$, so by the monotonicity of our speed $\kappa_{\epsilon}, \kappa_{\epsilon}\left(W\left(p_{0}\right)\right) \geq \kappa_{\epsilon}\left(W\left(q_{0}\right)\right)$. The maximum principle tells us that $d_{\min }(t)$ is non-decreasing and therefore the distance between the images of $F_{\epsilon}$ and $\psi_{\epsilon}$ is non-decreasing. Hence, they stay disjoint in time. As a consequence of that, our hypersurfaces $F_{\epsilon}(\cdot, t)$ stay enclosed by the sphere $\psi_{0}$ for all times of their existence (since $\psi_{\epsilon}(\cdot, t)$ are enclosed by $\psi_{0}$ ) and therefore $\left|F_{\epsilon}\right|(\cdot, t) \leq C$ for a uniform constant $C$.

The above bound implies that $\left\langle F_{\epsilon}, \nu\right\rangle \leq C$, for a uniform in $\epsilon$ constant $C$ and all $t \in\left[0, T_{\epsilon}\right)$. This together with (3.7) yield to (3.8).

\subsection{Curvature pinching estimates for the $\epsilon$-flow. Define}

$$
\mathcal{F}_{\epsilon}:=\left\langle F_{\epsilon}, \nu\right\rangle+2 t \kappa_{\epsilon} .
$$

Notice that division by $\mathcal{F}_{\epsilon}$ makes sense since by Lemma 3.3, $\left(\mathcal{F}_{\epsilon}\right)_{\min }$ is increasing in time and $\left(\mathcal{F}_{\epsilon}\right)_{\min }(0) \geq \delta>0$ due to star-shapedness. As we showed above, 
$\sup _{\Sigma_{t}}\left|F_{\epsilon}\right| \leq C$ for a uniform constant $C$. Rewrite the evolution equation for $H$ from Lemma 2.3 in the form

$$
\frac{\partial}{\partial t} H=\mathcal{L}_{\epsilon}(H)+\ddot{\kappa}_{\epsilon}(\nabla W, \nabla W)+\dot{\kappa}_{\epsilon}\left(W^{2}\right) H
$$

where $W$ is the Weingarten map and

$$
\ddot{\kappa}_{\epsilon}(\nabla W, \nabla W)=\frac{\partial^{2} \kappa_{\epsilon}}{\partial h_{q}^{p} \partial h_{m}^{l}} \nabla^{i} h_{q}^{p} \nabla_{j} h_{m}^{l} \quad \text { and } \quad \dot{\kappa}_{\epsilon}\left(W^{2}\right)=\frac{\partial \kappa_{\epsilon}}{\partial h_{m}^{l}} h_{p}^{l} h_{m}^{p} .
$$

Then, by direct computation we have

$$
\frac{\partial}{\partial t}\left(\frac{H}{\mathcal{F}_{\epsilon}}\right)=\mathcal{L}_{\epsilon}\left(\frac{H}{\mathcal{F}_{\epsilon}}\right)+\frac{2}{\mathcal{F}_{\epsilon}} \dot{\kappa}_{\epsilon}\left(\nabla \mathcal{F}, \nabla \frac{H}{\mathcal{F}_{\epsilon}}\right)+\frac{1}{2 \mathcal{F}_{\epsilon}} \operatorname{trace}_{g} \ddot{\kappa}_{\epsilon}(\nabla W, \nabla W) .
$$

By (2.9), the last term in this equation is negative. Hence, by the maximum principle, the supremum of $H / \mathcal{F}_{\epsilon}$ is decreasing. In particular, we have:

Lemma 3.6. Assume that $\Sigma_{t}^{\epsilon}$ is a solution of $\left(\underline{\left.\mathrm{HMCF}_{\epsilon}\right)}\right.$ on $\left[0, T_{\epsilon}\right)$ with $\Sigma_{0}$ as in Theorem 2.1, Then,

$$
\sup _{\Sigma_{t}^{\epsilon} \times[0, \tau)} \frac{H}{\left\langle F_{\epsilon}, \nu\right\rangle+2 t \kappa_{\epsilon}} \leq C, \quad \text { on }\left[0, T_{\epsilon}\right)
$$

for a uniform constant $C$ that depends only on $\Sigma_{0}$.

Denote by $\lambda_{1}, \lambda_{2}$ the two principal curvatures of the surface $\Sigma_{t}^{\epsilon}$ at some time $t$ and point $P$.

Lemma 3.7. If there is some time $t_{0}$ so that $\liminf _{t \rightarrow t_{0}} H(\cdot, t)=0$, then

$$
\liminf _{t \rightarrow t_{0}}\left(\lambda_{1}^{2}+\lambda_{2}^{2}\right)=0
$$

Proof. Assume $\liminf \operatorname{it}_{t \rightarrow t_{0}} H(\cdot, t)=0$. We distinguish the following two cases:

(i) $\lambda_{1}>0$ and $\lambda_{2} \geq 0$. In this case (3.12) immediately follows.

(ii) $\lambda_{1}>0$ and $\lambda_{2}<0$. By Lemma 3.3.

$$
\kappa_{\epsilon}:=\frac{G}{H}+\epsilon H \geq-C
$$

uniformly in time, which implies

$$
\lambda_{1}\left|\lambda_{2}\right| \leq C H+\epsilon H^{2} .
$$

Since $\liminf _{t \rightarrow t_{0}} H=0$, at least for one of the two principal curvatures must tend to zero, i.e.

$$
\liminf _{t \rightarrow t_{0}}\left|\lambda_{i}\right|=0 .
$$

Since $\liminf \operatorname{it}_{t \rightarrow t_{0}} H=0$, (3.12) readily follows. 
Lemma 3.8. There exist uniform (in time $t$ and $\epsilon$ ) constants $C>0$ and $\epsilon_{0}>0$, such that for every $0<\epsilon \leq \epsilon_{0}$, if $\lambda_{2} \leq 0$ at $P$, then

$$
\lambda_{1} \leq C
$$

Proof. Since $\lambda_{2} \leq 0$, we have $G / H \leq 0$. Hence, from (3.11) and the bound $\left|\left\langle F_{\epsilon}, \nu\right\rangle\right| \leq C_{0}$, for a uniform in time constant $C_{0}$, we conclude that

$$
H \leq C+C \epsilon H
$$

for a constant $C$ that depends only on the initial data. We conclude that for $\epsilon \leq \epsilon_{0}$, with $\epsilon_{0}$ sufficiently small depending only on the initial data $\Sigma_{0}$, we have

$$
H:=\lambda_{1}+\lambda_{2} \leq C
$$

from which the desired bound on $\lambda_{1}$ follows with the aid of the previous lemma.

Lemma 3.9. There exist uniform (in $t$ and $\epsilon$ ) constants $C>0$ and $\epsilon_{0}>0$, such that for every $0<\epsilon \leq \epsilon_{0}$ we have

$$
\lambda_{2} \geq-C \text {. }
$$

Proof. Assume that $\lambda_{2}<0$ (otherwise the bound is obvious). Then, $\lambda_{1}>0$ (since $\left.H=\lambda_{1}+\lambda_{2}>0\right)$ and by Lemma 3.5, we have

$$
\kappa_{\epsilon}:=\frac{G}{H}+\epsilon H \geq-C
$$

for a uniform in time constant $C$. Also, by the previous lemma $H \leq \lambda_{1} \leq C$. Hence,

$$
\left|\lambda_{2}\right| \leq C(1+\epsilon) \frac{\lambda_{1}+\lambda_{2}}{\lambda_{1}} \leq \tilde{C} .
$$

Remark 3.10. Lemma 3.8 implies that if the flow terminates because of the blowing up of the second fundamental form, that could only happen in the convex region of $\Sigma_{t}^{\epsilon}$ where $\lambda_{1} \geq 0, \lambda_{2} \geq 0$.

Lemma 3.11. There exist uniform (in time $t$ and $\epsilon$ ) constants $C>0, C_{0}>0$ and $\epsilon_{0}$, such that for every $\epsilon \geq \epsilon_{0}$ if $\lambda_{1} \geq C_{0}$ at $P$, then $\lambda_{2}>0$ at $P$ and

$$
1 \leq \frac{\lambda_{1}}{\lambda_{2}} \leq C .
$$


Proof. From the previous lemma, $\lambda_{2}>0$ if $C_{0}$ is chosen sufficiently large. Hence, from the bound (3.11) we conclude

$$
\begin{aligned}
\left(\lambda_{1}+\lambda_{2}\right)^{2} & \leq C_{1}\left(\lambda_{1}+\lambda_{2}\right)+2 T_{\epsilon}\left[\lambda_{1} \lambda_{2}+\epsilon\left(\lambda_{1}+\lambda_{2}\right)\right] \\
& \leq \tilde{C}_{1}\left(\lambda_{1}+\lambda_{2}\right)+\tilde{C}_{2} \lambda_{1} \lambda_{2}
\end{aligned}
$$

for some uniform in $\epsilon$ and $t$ constants $\tilde{C}_{1}$ and $\tilde{C}_{2}$. By taking $C_{0}$ sufficiently large, we can make

$$
\left(\lambda_{1}+\lambda_{2}\right)^{2}-C_{1}\left(\lambda_{1}+\lambda_{2}\right) \geq \frac{1}{2}\left(\lambda_{1}+\lambda_{2}\right)^{2} .
$$

Hence, (3.14) implies the bound

$$
\lambda_{1}^{2}+\lambda_{2}^{2} \leq 2 \tilde{C}_{2} \lambda_{1} \lambda_{2}
$$

from which the desired estimate readily follows.

To facilitate future references we combine the previous three lemmas in the following proposition:

Proposition 3.12. There exist $\epsilon_{0}>0$ and positive constants $C_{1}, C_{2}$, uniform in $0<\epsilon<\epsilon_{0}$ and $t$, so that for every $0<\epsilon<\epsilon_{0}$, we have
i. $\lambda_{2} \geq-C_{1}$, and
ii. $\lambda_{1} \leq C_{1} \lambda_{2}+C_{2}$.

In the proof of Theorem 3.1 we will also need the following bound.

Lemma 3.13. There is a uniform constant $C$, independent of $\epsilon$ and $t$ so that

$$
\int_{\Sigma_{t}^{\epsilon}} H^{2} d \mu_{t} \leq C
$$

Proof. We begin by noticing that that $\int_{\Sigma_{t}^{\epsilon}} G d \mu_{t}$ is a topological invariant, equal to $2 \pi \chi$, where $\chi$ is the Euler charactersistic of $\Sigma_{0}$. Since $\chi=2$ we then have

$$
\int_{\Sigma_{t}^{\epsilon}} G d \mu_{t}=4 \pi
$$

At any point we can choose the coordinates in which the second fundamental form is diagonal, with eigenvalues $\lambda_{1}$ and $\lambda_{2}$ as before and $\lambda_{1} \geq \lambda_{2}$. By Lemma 3.12 we have $\lambda_{1} \leq C_{1} \lambda_{2}+C_{2}$ which gives the inequality

$$
G:=\lambda_{1} \lambda_{2} \geq \frac{1}{C_{1}} \lambda_{1}^{2}-\frac{C_{2}}{C_{1}} \lambda_{1} .
$$

Using Cauchy-Scwartz we conclude the bound

$$
\lambda_{1} \lambda_{2} \geq \tilde{C}_{1} \lambda_{1}^{2}-\tilde{C}_{2}
$$


THE HARMONIC MEAN CURVATURE FLOW OF NONCONVEX SURFACES IN $\mathbb{R}^{3} \quad 21$ where $\tilde{C}_{1}, \tilde{C}_{2}$ are some uniform constants independent of $\epsilon$ and time. This yields to the estimate

$$
|A|^{2}=\lambda_{1}^{2}+\lambda_{2}^{2} \leq C_{1} G+C_{2}
$$

which after integrated over $\Sigma_{t}^{\epsilon}$ implies the bound

$$
\int_{\Sigma_{t}^{\epsilon}}|A|^{2} d \mu_{t} \leq C_{1} \int_{\Sigma_{t}^{\epsilon}} G d \mu_{t}+C_{2} \mu_{t}\left(\Sigma_{t}^{\epsilon}\right)
$$

with $\mu_{t}\left(\Sigma_{t}^{\epsilon}\right)$ denoting, as above, the surface area of $\Sigma_{t}^{\epsilon}$. By (3.1), $\mu_{t}\left(\Sigma_{t}^{\epsilon}\right) \leq \mu_{0}\left(\Sigma_{0}\right)$, where $\mu_{0}\left(\Sigma_{0}\right)$ denotes the surface area of $\Sigma_{0}$. Hence, the lemma readily follows from (3.15).

3.3. The proof of Theorem 3.1. Having all the ingredients from the previous sections we will finish the proof of Theorem 3.1 .

Proof of Theorem 3.1. Fix an $\epsilon$ and let $T=T_{\epsilon}$ be a maximal time up to which the flow exists. To simplify the notation we will omit the $\epsilon$-scripts from everything, including $T_{\epsilon}$ and the surface $\Sigma_{t}^{\epsilon}$, denoting them by $T$ and $\Sigma_{t}$ respectively. Because of Proposition 3.2, the second fundamental form blows up at time T. Hence, there is a sequence of $t_{i} \rightarrow T$ and $p_{i} \in \Sigma_{t_{i}}$ so that

$$
Q_{i}:=|A|\left(p_{i}, t_{i}\right)=\max _{t \in\left[0, t_{i}\right]} \max _{\Sigma_{t_{i}}}|A|\left(\cdot, t_{i}\right) \rightarrow \infty, \quad \text { as } i \rightarrow \infty .
$$

Consider the sequence $\tilde{\Sigma}_{t}^{i}$ of rescaled solutions defined by

$$
\tilde{F}_{i}(\cdot, t):=Q_{i}\left(F\left(\cdot, t_{i}+\frac{t}{Q_{i}^{2}}\right)-p_{i}\right) .
$$

Notice that under the above rescaling all points $p_{i}$ are shifted to the origin. If $g, H$ and $A:=\left\{h_{j k}\right\}$ are the induced metric, the mean curvature and the second fundamental form of $\Sigma_{t}$, respectively, then the corresponding rescaled quantities are given by

$$
\tilde{g}_{i}=Q_{i}^{2} g, \quad \tilde{H}_{i}=\frac{H}{Q_{i}}, \quad\left|\tilde{A}_{i}\right|^{2}=\frac{|A|^{2}}{Q_{i}^{2}} .
$$

Consider a sequence of rescaled solutions $\tilde{\Sigma}_{t}^{i}$. They have a property that

$$
\max _{\tilde{\Sigma}_{t}^{i}}\left|\tilde{A}_{i}\right| \leq 1, \text { for } t \in[-1,0] \text { and }\left|\tilde{A}_{i}\right|(0,0)=1 .
$$

The above uniform estimates on the second fundamental form yield uniform higher order estimates on $\tilde{F}_{i}(\cdot, t)$ and the Theorem of Arzela-Ascoli gives us a uniformly 
convergent subsequence $\tilde{F}_{i_{k}}(\cdot, t)$ on compact subsets, converging to a smooth $\tilde{F}(\cdot, t)$ for every $t \in[0,1]$. Notice that

$$
\tilde{\kappa}_{i}=\frac{\tilde{G}_{i}}{\tilde{H}_{i}}+\epsilon \tilde{H}_{i}=\frac{\lambda_{1} \lambda_{2}}{Q_{i}\left(\lambda_{1}+\lambda_{2}\right)}+\epsilon \frac{\lambda_{1}+\lambda_{2}}{Q_{i}}
$$

and therefore by Proposition 3.12

$$
\left|\tilde{\kappa}_{i}\right| \leq \begin{cases}\frac{C}{Q_{i}} & \text { if } \lambda_{1}, \lambda_{2}<<Q_{i} \\ C, & \text { if } \lambda_{1}, \lambda_{2} \sim Q_{i}\end{cases}
$$

since $\lambda_{2} \geq-C$ and $\lambda_{1}$ is big, comparable to the rescaling constant $Q_{i}$, if and only if $\lambda_{2}$ is big and comparable to $Q_{i}$ (both $\lambda_{1}$ and $\lambda_{2}$ are computed at time $t_{i}+t / Q_{i}^{2}$ ). This implies that $\tilde{F}(\cdot, t)$ solves $\frac{\partial}{\partial t} \tilde{F}(\cdot, t)=-\tilde{\kappa}_{\epsilon} \nu$, where

$$
\tilde{\kappa}_{\epsilon}= \begin{cases}0, & \text { if } \tilde{\lambda}_{1}=0, \tilde{\lambda}_{2}=0 \\ \frac{\tilde{\lambda}_{1} \tilde{\lambda}_{2}}{\tilde{\lambda}_{1}+\tilde{\lambda}_{2}}+\epsilon\left(\tilde{\lambda}_{1}+\tilde{\lambda}_{2}\right), & \text { if } \tilde{\lambda}_{1}>0, \tilde{\lambda}_{2}>0 .\end{cases}
$$

By Proposition 3.12 there are uniform constants $C_{1}, C_{2}$ so that

$$
\lambda_{1} \leq C_{1} \lambda_{2}+C_{2}
$$

which holds uniformly on $\Sigma_{t}$, for all $t \geq 0$ for which the flow exists, which after rescaling yields

$$
\tilde{\lambda}_{1}^{i} \leq C_{1} \tilde{\lambda}_{2}^{i}+\frac{C_{2}}{Q_{i}}
$$

The previous estimate implies that the limiting surface (which we denote by $\tilde{\Sigma}_{0}$ ) is convex (possibly not strictly convex). There are two possibilities for $\tilde{\Sigma}_{0}$ : either it is a flat plane or it is a non-flat complete weakly convex smooth hypersurface in $\mathbb{R}^{3}$. Let $\tilde{F}_{0}$ be a smooth embedding of $\tilde{\Sigma}_{0}$ into $\mathbb{R}^{3}$. Due to our rescaling, the norm of the second fundamental form of rescaled surfaces is 1 at the origin and therefore $\tilde{\Sigma}_{0}$ is not a plane, but is strictly convex at least somewhere. It has the property that

$$
\sup _{\tilde{\Sigma}_{0}}|\tilde{A}| \leq C .
$$

By the results in 9 there is a smooth complete solution $\bar{\Sigma}_{t}$ to the mean curvature flow

$$
\begin{cases}\frac{\partial}{\partial t} \bar{F}(p, t) & =-\bar{H} \nu(p, t), \quad p \in \bar{\Sigma}_{t}, t>0 \\ \bar{F}(p, 0) & =\tilde{F}_{0}\end{cases}
$$


The results in [9] (see Theorems 2.1, 2.3, 3.1 and 3.4, which provide with curvature estimates and are of local nature) imply that the curvature of $\bar{\Sigma}_{t}$ stays uniformly bounded for some short time $t \in\left[0, T_{0}\right)$. The evolution for $\bar{H}$ along the mean curvature flow is given by

$$
\frac{\partial}{\partial t} \bar{H}=\Delta \bar{H}+\bar{H}|\bar{A}|^{2} .
$$

As in [9], due to the curvature bounds, the mean curvature $\bar{H}$ satisfies the conditions of Theorem 4.3 in 9 (the maximum principle for parabolic equations on complete hypersurfaces) and therefore nonnegative mean curvature is preserved along the flow. This together with the strong maximum principle implies that if $\bar{H}$ is not identically zero at $t=0$, then it becomes strictly positive at $t>0$. We also know that $\bar{\Sigma}_{0}$ satisfies $\bar{\lambda}_{1} \leq C \bar{\lambda}_{2}$ for a uniform constant $C$, which follows from (3.19) after taking the limit as $i \rightarrow \infty$. Since we are assuming $\bar{\lambda}_{1} \geq \bar{\lambda}_{2}$ this can be written as

$$
\bar{h}_{i j} \geq \eta \bar{H} \bar{g}_{i j}
$$

for some uniform constant $\eta>0$ and we will say the second fundamental form of $\bar{\Sigma}$ is $\eta$-pinched.

By the curvature bounds, the maximum principle for complete hypersurfaces and the evolution for $\bar{h}_{i j}-\eta \bar{H} \bar{g}_{i j}$ it follows that the pinching estimate (3.21) is preserved by the mean curvature flow (as in [14]). In particular, this implies that $\bar{h}_{i j}$ is strictly positive definite, which means $\bar{\Sigma}_{t}$ is strictly convex for $t>0$. The result of R. Hamilton in [13] states that a smooth strictly convex and complete hypersurface with its second fundamental form $\eta$-pinched must be compact. Hence, it follows that $\bar{\Sigma}_{t}$ has to be compact for $t>0$. In this case, the initial data $\tilde{\Sigma}_{0}$ has to be compact as well.

We recall that $\tilde{\Sigma}_{0}$ is the limit of the hyper-surfaces $\tilde{\Sigma}_{0}^{i}$ which are obtained via re-scaling from the surfaces $\Sigma_{t_{i}}$. Hence, since $\tilde{\Sigma}_{0}$ is compact, there are constants $i_{0}, C$ so that for $i \geq i_{0}$, we have

$$
\operatorname{diam}\left(\Sigma_{t_{i}}\right)<\frac{C}{Q_{i}} \rightarrow 0 \text { as } i \rightarrow \infty,
$$

and therefore $\Sigma_{t_{i}} \rightarrow\{\bar{p}\}$.

Claim 3.14. For any point $q \in \mathbb{R}^{3}$, we have

$$
\frac{\partial}{\partial t}|F-q|^{2}=\mathcal{L}_{\epsilon}\left(|F-q|^{2}\right)-2 \frac{|A|^{2}}{H^{2}} .
$$

Proof. Follows by a simple computation. 
By Claim 3.14, $|F-\tilde{p}|_{\max }(t)$ is decreasing along $\left(\overline{\mathrm{HMCF}_{\epsilon}}\right)$ and therefore

$$
\Sigma_{t} \rightarrow\{\tilde{p}\}, \quad \text { as } t \rightarrow T
$$

which implies that the surface $\Sigma_{t}$ shrinks to a point as as $t \rightarrow T$. Hence, $\mu_{t}\left(\Sigma_{t}\right) \rightarrow 0$ as $t \rightarrow T$. It follows by (3.1) that $T$ must be given by (3.3).

\section{Passing to the Limit $\epsilon \rightarrow 0$}

We will assume in this section that $\Sigma_{t}^{\epsilon}$ are solutions of the flow $\left(\mathrm{HMCF}_{\epsilon}\right)$ which satisfy the condition (3.4) uniformly in $\epsilon$, with $T_{\epsilon}$ given by (3.3). We shall show that we can pass to the limit $\epsilon \rightarrow 0$ to obtain a solution of the HMCF which is defined up to time

$$
T:=\lim _{\epsilon \rightarrow 0} T_{\epsilon}=\frac{\mu_{0}\left(\Sigma_{0}\right)}{4 \pi} .
$$

The key result is the following uniform bound on the second fundamental form $A$ of $\Sigma_{\epsilon}$.

Proposition 4.1. Under assumption (3.4), for any $\tau<T$, there is a uniform constant $C=C(\tau)$ so that

$$
\max _{\Sigma_{t}^{\epsilon}}|A|(\cdot, t) \leq C, \quad \forall \epsilon>0 \quad \text { and } \quad \forall t \in[0, \tau]
$$

where $A$ denotes the second fundamental form of the surface $\Sigma_{t}^{\epsilon}$.

Proof. Assume there is $\tau<T$ for which (4.1) doesn't hold. Then, there exist sequences $t_{i} \rightarrow \tau, \epsilon_{i} \rightarrow 0$ and $p_{i} \in \Sigma_{t_{i}}^{\epsilon_{i}}$ so that

$$
Q_{i}:=|A|\left(p_{i}, t_{i}\right)=\max _{\Sigma_{t}^{\epsilon_{i}} \times\left[0, t_{i}\right]}|A| \rightarrow \infty \text { as } j \rightarrow \infty .
$$

Consider, as before, the rescaled sequence of solutions $\tilde{\Sigma}_{t}^{i}$ defined by the immersions $\tilde{F}_{i}(\cdot, t): M^{2} \rightarrow \mathbb{R}^{3}$

$$
\tilde{F}_{i}(\cdot, t):=Q_{i}\left(F_{\epsilon_{i}}\left(\cdot, t_{i}+\frac{t}{Q_{i}^{2}}\right)-p_{i}\right) .
$$

Due to our rescaling, the second fundamental form of rescaled surfaces is uniformly bounded in $i$. This uniform estimates on the second fundamental form yield uniform $C^{2}$-bounds on $\tilde{F}_{i}(\cdot, 0)$ and the Theorem of Arzela-Ascoli gives us a uniformly convergent subsequence on compact subsets, converging in the in $C^{1,1}$-topology to a $C^{1,1}$ surface $\tilde{\Sigma}$ defined by the immersion $\tilde{F}$.

By Lemma 3.12 there are uniform constants $C_{1}, C_{2}$ so that the estimate

$$
\lambda_{1} \leq C_{1} \lambda_{2}+C_{2}
$$


holds uniformly on $\Sigma_{t}^{\epsilon}$, for all $t \geq 0$ for which the flow exists, and all $\epsilon$, which after rescaling yields to the estimate

$$
\tilde{\lambda}_{1}^{i} \leq C_{1} \tilde{\lambda}_{2}^{i}+\frac{C_{2}}{Q_{i}}
$$

Hence, the limiting surface $\tilde{\Sigma}$ is convex. There are two possibilities for $\tilde{\Sigma}$, either it is a flat plane, or it is a complete convex $C^{1,1}$-hypersurface.

Due to our rescaling, the curvatures of the rescaled surfaces $\tilde{\Sigma}_{t}^{i}$ are uniformly bounded in $i$. This in particular implies a uniform local Lipshitz condition on $\tilde{F}_{i}\left(M^{2}, 0\right)$. This means that there are fixed numbers $r_{0}$ and $C_{0}$ so that for every $q \in \tilde{F}_{i}\left(M^{2}\right), \tilde{F}_{i}\left(U_{r_{0}, q}\right)$ (where $U_{r_{0}, q}$ is a component of $\tilde{F}_{i}^{-1}\left(B_{r_{0}}\left(\tilde{F}_{i}(q)\right)\right.$ ) containing $q$, and $B_{r_{0}}$ is a ball of radius $r_{0}$ in $\mathbb{R}^{3}$ ) can be written as the graph of a Lipshitz function over a hyperplane in $\mathbb{R}^{3}$ through $\tilde{F}_{i}(q)$ with Lipshitz constant less than $C_{0}$. Notice that both $C_{0}$ and $r_{0}$ are independent of $i$, they both depend on a uniform upper bound on the second fundamental form. This means the limiting surface $\tilde{\Sigma}$ will satisfy a uniform local Lipshitz condition.

Lemma 4.2. The limiting hypersurface $\tilde{\Sigma}$ is not a plane.

Proof. Assume that the limiting hypersurface $\tilde{\Sigma}$ is a plane. Then, for each $i$ we can write $\tilde{\Sigma}_{i}$ in a neighbourhood which is a ball $B(0,1)$ of radius 1 around the origin as a graph of a $C^{2}$-function $\tilde{u}_{i}$, over some hyperplane $\mathcal{H}_{i}$. In particular, we can choose one that is tangent to $\tilde{\Sigma}_{i}$ at the origin. Then

$$
\tilde{h}_{j k}^{i}=\frac{D_{j k} \tilde{u}_{i}}{\left(1+\left|D \tilde{u}_{i}\right|^{2}\right)^{\frac{1}{2}}} .
$$

We can choose a coordinate system in each hyperplane so that the second fundamental form and also $D^{2} u_{i}$ are diagonal at the origin. The function $u_{i}$ is a height function that measures the distance of our surface from the hyperplane $\mathcal{H}_{i}$. We also have that $u_{i} \stackrel{C^{1,1}}{\rightarrow} \tilde{u}$ as $i \rightarrow \infty$ and $u_{i}(0)=0$ for all $i$. If $\tilde{\Sigma}$ were a plane then $\tilde{u} \equiv 0$ and $\left|D \tilde{u}_{i}\right| \equiv 0$ which would imply $|\tilde{u}|_{C^{1,1}} \equiv 0$. Take $\epsilon>0$ very small. Then there would exist $i_{0}$ so that for $i \geq i_{0},\left|u_{i}\right|_{C^{1,1}}<\epsilon$ on $B(0,1) \subset \tilde{\Sigma}_{i}$. Since we have (4.2), the last estimate would contradict the fact $\left|\tilde{A}_{i}\right|(0,0)=1$, that is valid by the way we rescaled our solution.

It follows from the previous lemma and the discussion above that $\tilde{\Sigma}$ is a complete convex, non-flat $C^{1,1}$-surface that satisfies $\tilde{\lambda}_{1} \leq C \tilde{\lambda}_{2}$, whenever those quantities are defined (since a surface is $C^{1,1}$, the principal curvatures are defined almost everywhere). Because of our uniform curvature estimates of the rescaled sequence, 
$\tilde{\Sigma}$ is a uniformly locally Lipschitz surface. By the results in [9] there is a solution $\bar{F}_{t}$ of the Mean Curvature flow (3.20) with initial data $\tilde{\Sigma}$ on some time interval $\left[0, T_{1}\right)$ and $\bar{F}_{t}$ is smooth for $t>0$. We can now carry out the same argument as in the proof of Theorem 3.1 to show that $\tilde{\Sigma}$ has to be compact. That would mean that for $j>>1$,

$$
\operatorname{diam}\left(\Sigma_{t_{j}}^{\epsilon_{j}}\right) \leq \frac{C}{Q_{j}} \quad \text { and } \quad \text { area }\left(\Sigma_{t_{j}}^{\epsilon_{j}}\right) \leq \frac{C}{Q_{j}}
$$

for a uniform constant $C$. Since $T_{j} \rightarrow \tau<T$, (4.3) and Lemma 3.13 contradict (3.1). This shows that (4.1) holds true, therefore finishing our proof.

We will now show that because of (4.1) we can pass along subsequences $\epsilon_{i} \rightarrow 0$ and show that the solutions $\Sigma_{t}^{\epsilon_{i}}$ converge to a solution $\Sigma_{t}$ of (

Observe first that since $\partial F_{\epsilon} / \partial t=-(\kappa+\epsilon H) \nu$, by Proposition 4.1 we have that $\left|\partial F_{\epsilon} / \partial t\right| \leq C$, uniformly in $\epsilon$. Hence, $F_{\epsilon}$ is uniformly Lipshitz in $t$. Combining this with Proposition 4.1 and the assumption (3.4), we conclude that for every $\tau<T$ there is a subsequence $\epsilon_{i} \rightarrow 0$ and a 1-parameter family of $C^{1,1}$ surfaces $F(\cdot, t)$, so that $F_{\epsilon_{i}} \rightarrow F$ in the $C^{1,1}$ norm, $\partial F_{\epsilon_{i}} / \partial t \rightarrow \partial F / \partial t$ in the weak sense and $F$ satisfies

$$
\frac{\partial F}{\partial t}=-\kappa \nu
$$

Due to (3.4) our solution has the property that

$$
\operatorname{ess~inf}_{\Sigma_{t} \times[0, T)} H \geq \delta .
$$

Claim 4.3. The limiting solution of (4.4) does not depend on the sequence $\epsilon_{i} \rightarrow 0$.

Proof. Consider the evolution of a surface $\Sigma_{t}$ by a fully-nonlinear equation of the form

$$
\frac{\partial F}{\partial t}=-\mathcal{F}\left(h_{i j}\right) \nu
$$

where $h_{i j}$ is the second fundamental form and $\mathcal{F}$ is a function of the eigenvalues of $\left\{h_{i j}\right\}$, which we denote by $\lambda_{1}, \lambda_{2}$ and assume that $\lambda_{1} \geq \lambda_{2}$. Let $\mu=\lambda_{2} / \lambda_{1}$ and take

$$
\mathcal{F}\left(\lambda_{2}, \mu\right)= \begin{cases}\frac{\lambda_{1} \lambda_{2}}{\lambda_{1}+\lambda_{2}}=\frac{\lambda_{2}}{1+\mu}, & \text { for } \mu \geq-\delta_{1} \\ \frac{\lambda_{2}}{1-\delta_{1}}, & \text { otherwise }\end{cases}
$$

which we can be written as

$$
\mathcal{F}\left(h_{i j}\right)= \begin{cases}\kappa, & \text { for } H g_{i j} \geq\left(1-\delta_{1}\right) h_{i j} \\ \frac{H+\sqrt{H^{2}-4 G}}{1-\delta_{1}}, & \text { otherwise. }\end{cases}
$$


THE HARMONIC MEAN CURVATURE FLOW OF NONCONVEX SURFACES IN $\mathbb{R}^{3} \quad 27$

We can also consider solutions of (1.1) in the viscosity sense (defined in [6] and [10]). In that case (4.6) can be written in the form

$$
u_{t}=\left\{\begin{array}{lc}
\frac{\operatorname{det}\left(D_{i}\left(\frac{D_{j} u}{D u}\right)\right)}{\operatorname{div}\left(\frac{D u}{D u}\right)}, & \text { when } \operatorname{div}\left(\frac{D u}{|D u|}\right) \delta_{i j} \geq \\
\frac{\operatorname{div}\left(\frac{D u}{|D u|}\right)+\sqrt{\operatorname{div}\left(\frac{D u}{|D u|}\right)-4 \operatorname{det}\left(D_{i}\left(\frac{D_{j} u}{|D u|}\right)\right)}}{1-\delta_{1}} & \text { otherwise. }
\end{array}\right.
$$

Equation (4.9) can be expressed as

$$
u_{t}+\mathcal{F}_{1}\left(t, \nabla u, \nabla^{2} u\right)=0
$$

with

$$
\mathcal{F}_{1}(t, p, X)=-\frac{|p| \operatorname{det}\left(X-\frac{p}{|p|} \otimes\left(X \cdot \frac{p}{|p|}\right)\right.}{\operatorname{trace}\left(\left(I-\frac{p}{|p|} \otimes \frac{p}{|p|}\right) \cdot X\right)}
$$

if

$$
I \cdot \operatorname{trace}\left(\left(I-\frac{p}{|p|} \otimes \frac{p}{|p|}\right) \cdot X\right) \geq\left(1-\delta_{1}\right)\left(X-X \cdot \frac{p}{|p|} \otimes \frac{p}{|p|}\right)
$$

and

$$
\begin{aligned}
\mathcal{F}_{1}(t, p, X)= & \frac{1}{1-\delta_{1}}\left(\frac { 1 } { | p | } \left(\operatorname{trace}\left(\left(\delta_{i j}-\frac{p}{|p|} \otimes \frac{p}{|p|}\right) X\right)\right.\right. \\
& +\sqrt{\frac{1}{|p|^{2}}\left[\left(\operatorname{trace}\left(\left(\delta_{i j}-\frac{p}{|p|} \otimes \frac{p}{|p|}\right) X\right)\right)^{2}-\frac{4}{|p|} \operatorname{det}\left(X-\frac{(X \cdot p)}{|p|} \otimes \frac{p}{|p|}\right)\right)}
\end{aligned}
$$

otherwise.

Notice that the lower bound (4.5) together with our curvature pinching estimates (that follow from the Proposition 3.12) imply that

$$
H g_{i j} \geq\left(1-\delta_{1}\right) h_{i j}
$$

for some $1>\delta_{1}>0$. This implies that we can view a solution to (4.4) as a solution to (4.6) with $F$ as in (4.8). The function $\mathcal{F}_{1}(t, p, X)$ is continuous on $(0, T) \times \mathbb{R}^{2} \backslash\{0\} \times S^{2 \times 2}$, it satisfies the conditions of Theorem 7.1 in [6] and (4.10) is a degenerate parabolic geometric equation in the sense of Definition 5.1 in [6]. Theorem 7.1 in [6] shows the uniqueness of viscosity solutions to (4.10). The $C^{1,1}$ solution on $[0, T)$ constructed above is a viscosity solution to (4.10) and by the uniqueness result it is the unique $C^{1,1}$ solution to (4.4). This means that the limiting solution of (4.4) does not depend on the sequence $\epsilon_{i} \rightarrow 0$. 


\section{RAdiAl CASE}

In this section we will employ the results from the previous section to completely describe the long time behaviour of (1.1) in the case of surfaces of revolution, $r=f(x, t)$ around the $x$-axis. For such a surface of revolution the two principal curvatures are given by

$$
\lambda_{1}=\frac{1}{f\left(1+f_{x}^{2}\right)^{\frac{1}{2}}} \quad \text { and } \quad \lambda_{2}=-\frac{f_{x x}}{\left(1+f_{x}^{2}\right)^{\frac{3}{2}}} .
$$

Therefore,

$$
H=\lambda_{1}+\lambda_{2}=\frac{-f f_{x x}+f_{x}^{2}+1}{f\left(1+f_{x}^{2}\right)^{\frac{3}{2}}}>0
$$

and

$$
G=\lambda_{1} \lambda_{2}=\frac{-f_{x x}}{f\left(1+f_{x}^{2}\right)^{2}}
$$

When the surface evolves by (1.1), $f(x, t)$ evolves by

$$
f_{t}=\frac{f_{x x}}{-f f_{x x}+f_{x}^{2}+1} .
$$

We will consider solutions $f(\cdot, t)$ on an interval $I_{t}=\left[a_{t}, b_{t}\right] \subset[0,1]$ such that $f\left(a_{t}, t\right)=f\left(b_{t}, t\right)=0, f>0$ and $\tilde{H}=-f f_{x x}+f_{x}^{2}+1>0$. From (5.1) we see that $\lambda_{1}>0$ and $\lambda_{2}$ changes its sign, depending on the convexity of $f$. The linearization of (5.2) around a point $f$ is

$$
\tilde{f}_{t}=\frac{1+f_{x}^{2}}{\tilde{H}^{2}} \tilde{f}_{x x}-\frac{2 f_{x} f_{x x}}{\tilde{H}^{2}} \tilde{f}_{x}+\frac{f_{x x}^{2}}{\tilde{H}^{2}} \tilde{f}
$$

which is uniformly parabolic when $\tilde{H}$ is away from zero, no matter what is the sign of the smaller eigenvalue $\lambda_{2}$.

Theorem 5.1. Assume that at time $t=0, \Sigma_{0}$ is a $C^{1,1}$ star-shaped surface of revolution $r=f(x, 0)$, for $x \in[0,1], f(0,0)=f(1,0)=0, f(\cdot, 0)>0$ and $H>0$. Then, the flow exists up to the maximal time

$$
T=\frac{\mu_{0}\left(\Sigma_{0}\right)}{4 \pi}
$$

when the surface $\Sigma_{t}$ contracts to a point. Moreover, the surface becomes strictly convex at time $t_{1}<T$ and asymptotically spherical at its extinction time $T$.

Since the equation is strictly parabolic when $\tilde{H}>0$, the short time existence of a smooth solution on some time interval $[0, \tau]$, follows by classical results. Having a smooth solution to (1.1) on $[0, \tau]$ implies that we have a smooth solution $f(\cdot, t)$ to (5.2). By the comparison principle, $f(x, t)$ is defined on $I_{t}=\left[a_{t}, b_{t}\right] \subset[0,1]$ 
THE HARMONIC MEAN CURVATURE FLOW OF NONCONVEX SURFACES IN $\mathbb{R}^{3} \quad 29$

and $f\left(a_{t}, t\right)=f\left(b_{t}, t\right)=0$. Since the surface is smooth and $H>0$ on $[0, \tau]$, the expressions for $\lambda_{1}$ and $\lambda_{2}$ in (5.1) yield to the bounds

$$
\limsup _{x \rightarrow a_{t}} f\left|f_{x}\right| \leq C_{1}(t) \quad \text { and } \quad \limsup _{x \rightarrow b_{t}} f\left|f_{x}\right| \leq C_{2}(t), \quad \text { for } 0 \leq t \leq \tau .
$$

In the next lemma we will show that the above bounds do not depend on the lower bound on $H$, but only on the initial data.

Lemma 5.2. Assume that the solution $f$ is smooth on $\left[0, t_{0}\right)$, for some $t_{0} \leq T$ and $H>0$ on $\left[0, t_{0}\right)$. Then, there exists a uniform constant $C$, depending only on initial data, so that

$$
f^{2} f_{x}^{2} \leq C, \quad \text { for all } t \in\left[0, t_{0}\right) .
$$

Proof. We will bound $f^{2} f_{x}^{2}$ from above by the maximum principle. Let us compute its evolution equation. We first compute the evolution of $f_{x}$ by differentiating (15.2) in $x$. We get

$$
\left(f_{x}\right)_{t}=\frac{f_{x x x}\left(1+f_{x}^{2}\right)-f_{x} f_{x x}^{2}}{\tilde{H}^{2}},
$$

which yields the following equation for $f_{x}^{2}$ :

$$
\begin{aligned}
\left(f_{x}^{2}\right)_{t} & =\frac{2 f_{x x x} f_{x}\left(1+f_{x}^{2}\right)-2 f_{x}^{2} f_{x x}^{2}}{\tilde{H}^{2}}=\frac{\left(\left(f_{x}^{2}\right)_{x x}-2 f_{x x}^{2}\right)\left(1+f_{x}^{2}\right)-2 f_{x}^{2} f_{x x}^{2}}{\tilde{H}^{2}} \\
& =\frac{\left(f_{x}^{2}\right)_{x x}\left(1+f_{x}^{2}\right)-4 f_{x}^{2} f_{x x}^{2}-2 f_{x x}^{2}}{\tilde{H}^{2}} .
\end{aligned}
$$

The function $f^{2}$ satisfies the equation

$$
\left(f^{2}\right)_{t}=\frac{\left(f^{2}\right)_{x x}-2 f_{x}^{2}}{\tilde{H}^{2}} .
$$

Combining the last two equations we obtain

$$
\begin{aligned}
\left(f^{2} f_{x}^{2}\right)_{t} & =\frac{\left(f_{x}^{2}\right)_{x x}\left(1+f_{x}^{2}\right)-4 f_{x}^{2} f_{x x}^{2}-2 f_{x x}^{2}}{\tilde{H}^{2}} f^{2}+2 \frac{f_{x x} f f_{x}^{2}}{\tilde{H}^{2}} \\
& =\frac{\left(f^{2} f_{x}^{2}\right)_{x x}\left(1+f_{x}^{2}\right)}{\tilde{H}^{2}}-\frac{\left(1+f_{x}^{2}\right)\left(\left(f^{2}\right)_{x x} f_{x}^{2}-2\left(f_{x}^{2}\right)_{x}\left(f^{2}\right)_{x}\right)}{\tilde{H}^{2}}+2 \frac{f_{x x} f f_{x}^{2}}{\tilde{H}^{2}} .
\end{aligned}
$$

Let $t<t_{0}$. We distinguish the following two cases:

Case 1. The $\left(f^{2} f_{x}^{2}\right)_{\max }(t)$ is attained in the interior of $\left(a_{t}, b_{t}\right)$. Then, at that point $\left(f^{2} f_{x}^{2}\right)_{x}=0$, which implies (since $f(\cdot, t)>0$ in the interior) that

$$
f_{x}^{3}=-f f_{x} f_{x x} .
$$


Hence, the maximum principle implies the differential inequality

$$
\begin{aligned}
\frac{d}{d t}\left(f^{2} f_{x}^{2}\right)_{\max }(t) & \leq-\frac{1+f_{x}^{2}}{\tilde{H}^{2}}\left(\left(f^{2}\right)_{x x} f_{x}^{2}-2\left(f_{x}^{2}\right)_{x}\left(f^{2}\right)_{x}\right)+2 \frac{f_{x x} f f_{x}^{2}}{\tilde{H}^{2}} \\
& =-8 \frac{\left(1+f_{x}^{2}\right) f_{x}^{4}}{\tilde{H}^{4}}-2 \frac{f_{x}^{4}}{\tilde{H}^{2}} \leq 0 .
\end{aligned}
$$

Case 2. The $\left(f^{2} f_{x}^{2}\right)_{\max }(t)$ is attained at one of the tips $\left\{a_{t}, b_{t}\right\}$. Assume it is attained at $a_{t}$. The point of the surface $\Sigma_{t}$ that arises from $x=a_{t}$ can be viewed as the interior point of $\Sigma_{t}$ around which our surface is convex. We can solve locally, around the point $x=a_{t}$ (say for $x \in\left[a_{t}, x_{t}\right]$ ) the equation $y=f(x, t)$ with respect to $x$, yielding to the map $x=g(y, t)$. Notice that $f f_{x}=y / g_{y}$ and that $x=a_{t}$ corresponds to $y=0$. Since $\left\{f(x, t) \mid x \in\left[a_{t}, x_{t}\right]\right\} \cup\{-f(x, t) \mid x \in$ $\left.\left[a_{t}, x_{t}\right]\right\}$ is a smooth curve, we have that $x=g(y, t)$ is a smooth graph for $y \in$ $\left[-f\left(x_{t}, t\right), f\left(x_{t}, t\right)\right]$. If $f^{2} f_{x}^{2}(\cdot, t)$ attains its maximum somewhere in $\left[a_{t}, x_{t}\right)$, then $y^{2} / g_{y}^{2}$ attains its maximum in the interior of $\left(-f\left(x_{t}, t\right), f\left(x_{t}, t\right)\right)$.

We will now compute the evolution of $y^{2} / g_{y}^{2}$ from the evolution of $f^{2} f_{x}^{2}$. Since

$$
f_{x}(x, t)=\frac{1}{g_{y}(y, t)}
$$

from the evolution of $f^{2} f_{x}^{2}$ we get

$$
\left(\frac{y^{2}}{g_{y}^{2}}\right)_{t}=\frac{\left(1+g_{y}^{2}\right)}{g_{y}^{2} \tilde{H}^{2}}\left(\frac{y^{2}}{g_{y}^{2}}\right)_{x x}-\frac{\left(1+g_{y}^{2}\right)}{g_{y}^{2} \tilde{H}^{2}}\left(\left(y^{2}\right)_{x x} \frac{1}{g_{y}^{2}}-2\left(\frac{1}{g_{y}^{2}}\right)_{x}\left(y^{2}\right)_{x}\right)+\frac{2 y_{x x} y}{g_{y}^{2} \tilde{H}^{2}} .
$$

By direct computation we have

$$
\left(\frac{1}{g_{y}^{2}}\right)_{x}=-\frac{2 g_{y y}}{g_{y}^{3}} \quad \text { and } \quad\left(y^{2}\right)_{x}=\frac{2 y}{g_{y}} \quad \text { and } \quad y_{x x}=-\frac{g_{y y}}{g_{y}^{3}}
$$

and

$$
\left(\frac{y^{2}}{g_{y}^{2}}\right)_{x x}=\left(\frac{y^{2}}{g_{y}^{2}}\right)_{y y}-\left(\frac{y^{2}}{g_{y}^{2}}\right)_{y} \frac{g_{y y}}{g_{y}^{3}} \quad \text { and } \quad\left(y^{2}\right)_{x x}=\frac{2}{g_{y}^{2}}-\frac{2 y g_{y y}}{g_{y}^{3}}
$$

Combining the above yields to

$$
\begin{aligned}
\left(\frac{y^{2}}{g_{y}^{2}}\right)_{t} & =\frac{\left(g_{y}^{2}+1\right)}{g_{y}^{2} \tilde{H}^{2}}\left(\frac{y^{2}}{g_{y}^{2}}\right)_{y y}-\left(\frac{y^{2}}{g_{y}^{2}}\right)_{y} \frac{g_{y y}\left(1+g_{y}^{2}\right)}{g_{y}^{5} \tilde{H}^{2}} \\
& -\frac{\left(1+g_{y}^{2}\right)}{g_{y}^{2} \tilde{H}^{2}}\left(\frac{2}{g_{y}^{4}}+2 \frac{y g_{y y}}{g_{y}^{5}}\right)-\frac{2 y g_{y y}}{g_{y}^{5} \tilde{H}^{2}}
\end{aligned}
$$


which can be re-written it as

$$
\begin{aligned}
\left(\frac{y^{2}}{g_{y}^{2}}\right)_{t} & =\frac{\left(g_{y}^{2}+1\right)}{g_{y}^{2} \tilde{H}^{2}}\left(\frac{y^{2}}{g_{y}^{2}}\right)_{y y}-\left(\frac{y^{2}}{g_{y}^{2}}\right)_{y} \frac{g_{y y}\left(1+g_{y}^{2}\right)}{g_{y}^{2} \tilde{H}^{5}} \\
& -\frac{\left(1+g_{y}^{2}\right)}{g_{y}^{2} \tilde{H}^{2}}\left(\frac{2}{g_{y}^{4}}+\frac{2 y^{2} g_{y} g_{y y}}{y g_{y}^{6}}\right)-\frac{2 y^{2} g_{y} g_{y y}}{y g_{y}^{6} \tilde{H}^{2}} .
\end{aligned}
$$

At the maximum point of $y^{2} / g_{y}^{2}$ we have

$$
y^{2} g_{y} g_{y y}=y g_{y}^{2}
$$

This together with the maximum principle applied to (5.8) yield to the differential inequality

$$
\frac{d}{d t}\left(\frac{y^{2}}{g_{y}^{2}}\right)_{\max }(t) \leq-\frac{4\left(1+g_{y}^{2}\right)}{g_{y}^{6} \tilde{H}^{2}}-\frac{2}{\tilde{H}^{2} g_{y}^{4}} \leq 0 .
$$

Estimates (5.7) and (5.9) imply that $\left(f^{2} f_{x}^{2}\right)(x, t) \leq C$, for all $x \in\left[a_{t}, b_{t}\right]$ and all $t \leq t_{0}$, where $C$ is a uniform constant independent of time. This finishes the proof of the lemma.

Corollary 5.3. Let $T=\mu_{0}\left(\Sigma_{0}\right) / 4 \pi$ be as in Theorem 5.1. Then, there exists a uniform constant $\delta$, depending only on the initial data, so that $H \geq \delta>0$, for all $t \in[0, T)$.

Proof. It is enough to show that if $H>0$ on $\left[0, t_{0}\right)$, then $H \geq \delta>0$ there. We recall that $\lambda_{1}=1 / f\left(1+f_{x}^{2}\right)^{1 / 2}$. Hence, the estimate (5.4) yields to the bound

$$
\lambda_{1} \geq c>0 \quad \text { on } \Sigma_{t}, \quad \text { for } t \in\left[0, t_{0}\right) .
$$

Since $H=\lambda_{1}+\lambda_{2}$, if $\lambda_{2} \geq 0$, then $H \geq \lambda_{1} \geq c$. If $\lambda_{2}<0$ and $H \leq c / 2$ (otherwise we are done) by (5.10) we have

$$
\lambda_{1}-\left|\lambda_{2}\right| \leq \frac{c}{2} \Rightarrow\left|\lambda_{2}\right| \geq \frac{c}{2} .
$$

Observe next that Lemma 3.5 implies the bound

$$
\frac{\lambda_{1}\left|\lambda_{2}\right|}{H} \leq C, \quad \text { for a uniform constant } C .
$$

Hence

$$
H \geq \frac{\lambda_{1}\left|\lambda_{2}\right|}{C} \geq \frac{c^{2}}{2 C}
$$

In any case, we have

$$
H \geq \min \left\{\frac{c}{2}, \frac{c^{2}}{2 C}\right\}
$$

which shows our lemma with $\delta:=\min \left\{\frac{c}{2}, \frac{c^{2}}{2 C}\right\}$. 
Lemma 5.4. Let $[0, T)$ be the maximal interval of existence of a solution to (1.1). Then, $\max _{\Sigma_{t}}|A|$ becomes unbounded as $t \rightarrow T$.

Proof. Assume that $\sup _{\Sigma_{t}}|A| \leq C$, for all $t \in[0, T)$ and write

$$
H=\frac{\tilde{H}}{f\left(1+f_{x}^{2}\right)^{3 / 2}}
$$

with $\tilde{H}=-f f_{x x}+f_{x}^{2}+1$. Then $H \leq C$ (since $|A|$ is bounded) and $H \geq \delta>0$ (by the previous result). Hence,

$$
c_{1} \leq \frac{f\left(f_{x}^{2}+1\right)^{3 / 2}}{\tilde{H}} \leq c_{2}
$$

which implies

$$
\frac{c_{1}}{f\left(1+f_{x}^{2}\right)^{1 / 2}} \leq \frac{1+f_{x}^{2}}{\tilde{H}} \leq \frac{c_{2}}{f\left(1+f_{x}^{2}\right)^{1 / 2}} .
$$

We can rewrite it as

$$
c_{1} \lambda_{1} \leq \frac{1+f_{x}^{2}}{\tilde{H}} \leq c_{2} \lambda_{1}
$$

which together with (5.10) and $|A| \leq C$ imply the bounds

$$
C_{1} \leq \frac{1+f_{x}^{2}}{\tilde{H}} \leq C_{2}
$$

for uniform constants $C_{1}, C_{2}$, for all $t \in[0, T)$. This means the linearization (5.3) of (5.2) is uniformly elliptic on time interval $[0, T)$. If our surface of revolution at time $t$ is given by an embedding $F(\Sigma, t)$, which is a solution to (1.1), $|A| \leq C$ implies $|F|_{C^{2}} \leq C$ on the time interval $[0, T)$ and the speed $|\kappa| \leq C$ (we will use the same symbol $C$ to denote different uniform constants). It is easy to see that $F(\cdot, t)$ converges to a continuous limit $F(\cdot, T)$ as $t \rightarrow T$, since

$$
\left|F\left(x, t_{1}\right)-F\left(x, t_{2}\right)\right| \leq \int_{t_{1}}^{t_{2}}|\kappa| d t \leq C\left|t_{1}-t_{2}\right| .
$$

Due to

$$
\left|\frac{\partial}{\partial t} g_{i j}\right|^{2}=\left|2 h_{i j} \kappa\right|^{2} \leq 4|A|^{2} \kappa^{2} \leq C
$$

and [12] we have that $F(\cdot, T)$ represents a surface. It is a $C^{1,1}$ surface of revolution $r=f(x, T)$ around the $x$-axis that comes as a limit as $t \rightarrow T$ of surfaces of revolution $r=f(x, t)$. Take $0<\epsilon<<b_{T}-a_{T}$ arbitrarily small. Consider $f(r, t)$ on $x \in\left[a_{t}+\epsilon, b_{t}-\epsilon\right]$, that is, away from the tips $x=a_{t}$ and $x=b_{t}$ where $f=0$ and $f_{x}$ becomes unbounded. Since our solution is $C^{1,1}, c_{1} \leq f(r, T) \leq c_{2}$ and $\left|f_{x}\right| \leq c_{3}$, at time $t=T$ and for $x \in\left[a_{T}+\epsilon, b_{T}-\epsilon\right]$, where $c_{1}, c_{2}, c_{3}$ all depend on $\epsilon$. Due 
to (5.12), equation (5.2) is uniformly parabolic and standard parabolic estimates yield

$$
|f(\cdot, T)|_{C^{k}} \leq C(\epsilon, k), \quad \text { for every } k>0 \text { and } x \in\left[a_{T}+\epsilon, b_{T}-\epsilon\right] .
$$

We can repeat the previous discussion to every $\epsilon>0$ to conclude that our surface $\Sigma_{T}$ is smooth for $x \in\left(a_{T}, b_{T}\right)$. By writing our surface locally as a graph $x=g(y, t)$ around the tips (at which our surface is strictly convex), we can show that our surface is smooth at the tips as well (similar methods to those discussed above apply in this case).

The same proof as the one for the flow $\left(\mathrm{HMCF}_{\epsilon}\right)$ which was presented in the previous section, shows that our radial surface $\Sigma_{t}$ shrinks to a point at $T=\frac{\mu_{0}\left(\Sigma_{0}\right)}{4 \pi}$, where $\mu_{0}\left(\Sigma_{0}\right)$ is the total area of $\Sigma_{0}$. In particular, this means $f(x, t) \rightarrow 0$ as $t \rightarrow T$.

We will show next that at some time $t_{1}<T$ the surface $\Sigma_{t_{1}}$ becomes strictly convex. This will follow from the next lemma.

Lemma 5.5. Assume that $f$ is a solution of the HMCF on $[0, T)$. Then, there exists a constant $c>0$, independent of $t$, such that $f(x, t) \geq c$, at all points $(x, t)$, with $0 \leq t<T$ and $f_{x x}(x, t) \geq 0$.

Proof. Fix $t<T$. Since our surface $\Sigma_{t}$ is convex around the tip $x=a_{t}$ we have $f_{x x} \leq 0$ there. Let $c_{t}$ be the largest number in $\left[a_{t}, b_{t}\right]$ so that $\Sigma_{t}$ is strictly convex for $x \in\left[a_{t}, c_{t}\right]$. If $c_{t}=b_{t}$, then $\Sigma_{t}$ is convex and we have nothing to show. Otherwise, $f_{x x}(x, t) \leq 0$ for $a_{t} \leq x \leq c_{t}$ and $f_{x x}(x, t)>0$ in $\left(c_{t}, c_{t}+\epsilon_{t}\right)$ for some $\epsilon_{t}>0$. Hence, $f_{x}(\cdot, t)$ is increasing in $x$, for $x \in\left(c_{t}, c_{t}+\epsilon_{t}\right)$.

Consider the function $f_{x}(\cdot, t)$ on the interval $x \in\left[c_{t}, b_{t}\right)$. From the above discussion and the fact that $\lim _{x \rightarrow b_{t}} f_{x}(x, t)=-\infty$, we conclude that the maximum

$$
M(t):=\max \left\{f_{x}(x, t), x \in\left[c_{t}, b_{t}\right]\right\}
$$

is attained in the interior of $\left[c_{t}, b_{t}\right]$. Recall the evolution equation for $f_{x}$ to be

$$
\left(f_{x}\right)_{t}=\frac{f_{x x x}\left(1+f_{x}^{2}\right)}{\tilde{H}^{2}}-\frac{f_{x} f_{x x}^{2}}{\tilde{H}^{2}} .
$$

Hence, assuming that $M(t) \geq 0$, the maximum principle implies that $M^{\prime}(t) \leq 0$. This shows that $f_{x}$ is uniformly bounded from above on $\left[c_{t}, b_{t}\right]$. Since a similar argument can be applied near the other tip $b_{t}$, we finally conclude that $\left|f_{x}\right|$ is uniformly bounded in the non-convex part (if it exists) away from the tips. 
We will now conclude the proof of the lemma. Assume that $f_{x x}(x, t) \geq 0$, which holds in a non-convex part of our evolving surface. At that point, we have

$$
\lambda_{2}:=-\frac{f_{x x}}{\left(1+f_{x}^{2}\right)^{3 / 2}} \leq 0 .
$$

Since $\lambda_{2} \leq 0$, Lemma 3.8 implies the bound

$$
\lambda_{1}:=\frac{1}{f\left(1+f_{x}^{2}\right)^{1 / 2}} \leq C
$$

which reduces to the the bound

$$
f \geq \frac{1}{C\left(1+f_{x}^{2}\right)^{1 / 2}} \geq \frac{1}{\tilde{C}}=: c
$$

in the non-convex part where $f_{x}^{2} \leq C$, uniformly in $t$. This finishes the proof of the lemma.

We will now conclude the proof of Theorem 5.1: Since $f(x, t) \rightarrow 0$ as $t \rightarrow T$, with $T=\frac{\mu_{0}\left(\Sigma_{0}\right)}{4 \pi}$, there is some time $t_{1}<T$ so that

$$
f(x, t)<\frac{c}{2}, \quad \text { for all } x \in\left[a_{t}, b_{t}\right]
$$

where $c>0$ is the constant taken from Lemma 5.5. Hence, by Lemma 5.5 the surface $\Sigma_{t}$ is convex for $t \geq t_{1}$. Since $H \geq \delta>0$ for all $t<T$, the surface $\Sigma_{t_{1}}$ is strictly convex. The result of Andrews in [1, implies that $\Sigma_{t}$ shrinks asymptotically spherically to a point as $t \rightarrow T$.

\section{REFERENCES}

[1] Andrews,B., Contraction of convex hypersurfaces in Euclidean space, Calc.Var. 2 (1994), $151-171$.

[2] B. Andrews. Contraction of convex hypersurfaces in Riemannian spaces J. Diff. Geometry. 39, no. 2, 407-431, 1994

[3] B. Andrews. Motion of hypersurfaces by Gauss curvature Pacific J. Math. 195, no. 1, 1-34, 2000 .

[4] B. Andrews. Pinching estimates and motion of hypersurfaces by curvature functions ArXiv: Math. DG /0402311

[5] M.C. Caputo, P. Daskalopoulos. Highly Degenerate Harmonic Mean Curvature flow, preprint.

[6] Y.-G.Chen, Y.Giga, S.Goto. Uniqueness and existence of viscosity solutions of generalized mean curvature flow equations. J.Diff.Geom. 33, 749-786, 1991.

[7] P.Daskalopoulos, R.Hamilton. Harmonic Mean Curvature flow on Surfaces of Negative Gaussian Curvature. Comm. Anal. Geom., 14 (2006), no. 5, 907-943. 
THE HARMONIC MEAN CURVATURE FLOW OF NONCONVEX SURFACES IN $\mathbb{R}^{3}$

[8] S.Diëter. Nonlinear degenerate Curvature flow for weakly convex hypersurfaces. Calculus of Variations and Partial Differential Equations, 22, 2: 229 - 251, 2005

[9] G.Huisken, K.Ecker. Interior estimates for hypersurfaces moving by mean curvature Invent. Math., 105: 547-569, 1991.

[10] L.C.Evans, J.Spruck. Motion of level sets by mean curvature I J. Diff. Geom. , 33, 635-681, 1991.

[11] C.Gerhardt. Flow of nonconvex hypersurfaces into spheres. J.Diff.Geom., 32, 299-314, 1990.

[12] R.Hamilton. Three-manifolds with positive Ricci curvature J.Diff.Geom. , 17, 255-306, 1982.

[13] R.Hamilton. Convex hypersurfaces with pinched second fundamental form Comm. Anal. Geom. , 2, 167-172, 1994.

[14] G.Huisken. Flow by mean curvature of convex hypersurfaces into spheres J. Diff. Geom. , 20, 237-266, 1984.

[15] N.V.Krylov. Nonlinear elliptic and parabolic equations of second order. D.Reidel, 1978.

[16] K.Smoczyk. Starshaped hypersurfaces and the mean curvature flow. Manuscripta Math., 95, 225-236, 1998.

[17] J.Urbas. On the expansion of starshaped surfaces by symmetric functions of their principal curvatures. Math.Z., 205, 355-372, 1990.

Department of Mathematics, Columbia University, New York, USA

E-mail address: pdaskalo@math.columbia.edu

Department of Mathematics, Columbia University, New York, USA

E-mail address: natasas@math.columbia.edu 